\title{
Geochemical signature of Eocene Kuh-e Dom shoshonitic dikes in NE Ardestan, Central Iran: implications for melt evolution and tectonic setting
}

\author{
Fatemeh SARJOUGHIAN ${ }^{1}$, Ali KANANIAN ${ }^{*}$, Michael HASCHKE², Jamshid AHMADIAN ${ }^{3}$ \\ ${ }^{1}$ School of Geology, College of Science, University of Tehran, Tehran, Iran; kananian@khayam.ut.ac.ir \\ ${ }^{2}$ Umwelt und Ingenieurtechnik GmbH, Zum Windkanal 21, 01109 Dresden, Germany \\ ${ }^{3}$ Department of Geology, Payame Noor University, Po BOX 19395-3697, Tehran, Iran \\ * Corresponding author
}

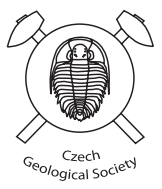

\begin{abstract}
The Late Eocene Kuh-e Dom composite intrusion forms a segment of the Urumieh-Dokhtar magmatic arc, which recorded syn- to post-collisional magmatism during the Alpine-Himalayan orogeny in central Iran. Numerous acid and intermediate-basic dikes intrude the composite intrusive complex of the arc segment and its host-rock assemblage. The silicic dikes of porphyric microgranite, porphyric microgranodiorite and aplite consist of quartz, K-feldspar, plagioclase (albite), biotite and rare amphibole. The dikes are of subaluminous composition with shoshonitic affinity. Trace-element patterns exhibit pronounced negative anomalies of $\mathrm{Nb}, \mathrm{Ta}, \mathrm{Ti}, \mathrm{P}$ and $\mathrm{Sr}$ together with positive anomalies of Cs, Th, $\mathrm{U}$ and La suggesting partial melting of a quartzo-feldspathic crustal source.

The intermediate-basic dikes with phonolite, basanite and trachyandesite chemical compositions typically contain pyroxene (diopside-augite) and plagioclase phenocrysts $\left(\mathrm{An}_{30-60}\right.$ and $\left.\mathrm{An}_{98}\right)$, calcic amphiboles (magnesiohornblendemagnesiohastingsite), magnesian biotites and alkali-feldspars $\left(\mathrm{Or}_{95}\right)$. The rocks show shoshonitic geochemical affinities. Low $\mathrm{Ba} / \mathrm{Rb}$ ratios and high $\mathrm{Rb} / \mathrm{Sr}$ ratios suggest that the primary dike melt originated by partial melting of a phlogopite-bearing lithospheric mantle, whereas LILE and LREE enrichment along with low $\mathrm{Nb} / \mathrm{Zr}$ and $\mathrm{Hf} / \mathrm{Sm}$ ratios and high $\mathrm{Ba} /$ $\mathrm{Nb}$ and $\mathrm{Rb} / \mathrm{Nb}$ ratios imply that these rocks formed at a convergent continental margin.

The acidic dikes were emplaced in a transitional syn-collisional subduction setting whereas the intermediate-basic dikes have developed from remnant melt batches after cessation of active subduction, mostly in the post-collisional setting.
\end{abstract}

Keywords: shoshonite dikes, collisional magmatism, mineral chemistry, Kuh-e Dom, petrogenesis, Iran Received: 29 March 2012; accepted: 26 November 2012; handling editor: E. Jelínek

\section{Introduction}

The Kuh-e Dom intrusion is a part of the UrumiehDokhtar magmatic arc, a 5-25 km wide and 2000 $\mathrm{km}$ long northwest-southeast trending magmatic belt in central Iran. It is aligned parallel with the suture of the collisional Zagros fold-thrust belt and reflects the subduction of the Neotethys as Africa moved northward relative to Eurasia. Igneous activity of this magmatic arc developed from the beginning of subduction in Jurassic (Alavi 1980) and reached a climax in the Middle Eocene (Berberian \& King 1981) with geochemical and petrological features similar to those of Andeantype magmatism (Berberian et al. 1982). The Neotethys is believed to have closed during Late Oligocene to Miocene (Mohajjel et al. 2003; Shahabpour 2007), initiating the collision between Arabia and Eurasia. Subduction-related magmatism in central Iran ranges from Cretaceous to Recent, but is dominated in volume by $50-35$ Ma old intermediate to silicic calc-alkaline and locally shoshonitic volcanic and intrusive rocks
(Berberian and King 1981; Berberian et al. 1982; Alavi and Mahdavi 1994).

The various dikes cutting the Urumieh-Dokhtar magmatic belt can be divided into two main groups: (1) silicic (porphyric microgranodiorites, porphyric microgranites and aplites), and (2) intermediate-basic (phonolite, basanite and trachyandesite). Both types exhibit shoshonitic nature which is inferred to represent a transitional stage between calc-alkaline and alkaline magmas (Liégeois et al. 1998). The dikes are characterized by high $\mathrm{K}_{2} \mathrm{O} / \mathrm{Na}_{2} \mathrm{O}$ ratios (>0.5), high $\mathrm{Na}_{2} \mathrm{O}+\mathrm{K}_{2} \mathrm{O}(>5$ wt. \%), LILE (e.g., $\mathrm{K}, \mathrm{Rb}, \mathrm{Sr}$ and $\mathrm{Ba}$ ), and generally low $\mathrm{TiO}_{2}$ contents $(<1.3$ wt. \%) similar to typical shoshonitic series (Joplin 1968; Morrison 1980).

Joplin (1965) suggested that shoshonitic rocks generally show $\mathrm{SiO}_{2}$ less than $70 \mathrm{wt}$ \% (from basaltic to trachytic rocks). On the other hand, Keller (1974) suggested that shoshonites should also include more silicic rocks such as dacite and rhyolite. Peccerillo and Taylor (1976) only regarded types with $\mathrm{SiO}_{2}<63 \mathrm{wt}$. \% as shoshonites. Morrison (1980), proposed shoshonites to be subdivided 
into basic $\left(\mathrm{SiO}_{2}<53\right.$ wt. \%), intermediate $\left(\mathrm{SiO}_{2}=53-63\right.$ wt. \%) and silicic $\left(\mathrm{SiO}_{2}>63\right.$ wt. \%).

It seems that the chemical variability of shoshonitic rocks is not completely understood in terms of source characteristics and geodynamic setting. Morrison (1980) suggested that shoshonites are restricted to subductionrelated tectonic settings, where they are generated during late-stage of arc evolution, typically after the low-K tholeiites and calc-alkaline rock series. Similarly, Müller and Groves (1997) concluded that shoshonites should be regarded as products of magmatic activity above subduction zones in continental arcs, post-collisional arcs, or in oceanic-arc settings where potassic rocks are related to the melting of phlogopite-bearing wedge.

The aim of this study is to contribute to the understanding of the acidic and intermediate-basic dikes in the Kuh-e Dom intrusion of the Urumieh-Dokhtar magmatic belt. More specifically, this contribution deals with the petrogenesis and tectonic setting of the dikes, by evaluating field, petrographic, whole-rock geochemical and mineral compositional data.

\section{Analytical methods}

The petrological variation and the crosscutting relations of the dikes were observed during field analysis. Both petrographic and geochemical analyses were conducted using the most representative and fresh samples from various lithologies in the studied intrusions. The thirteen samples were selected for whole-rock geochemical analysis and 90 points for microprobe analysis.

The mineral analyses were performed in the electron microprobe Laboratory at Naruto University, Japan (Jeol JXA-8800R) equipped by WDS detector using $15 \mathrm{kV}, 20$ $\mathrm{nA}$ and $20 \mathrm{~s}$ counting time and University of Oklahoma, Norman, USA (Cameca SX50) with five asynchronous wavelength-dispersive spectrometers and PGT PRISM 2000 energy-dispersive X-ray analyzer. Petrographic characterization was performed by backscattered electron imaging coupled with energy-dispersive $\mathrm{X}$-ray analysis using $20 \mathrm{kV}$ acceleration voltage and $20 \mathrm{nA}$ sample current. Chemical microanalysis was carried out by wavelength-dispersive spectrometry using $20 \mathrm{kV}$ acceleration voltage, $20 \mathrm{nA}$ beam current (measured at the Faraday cup), and $2 \mu \mathrm{m}$ spot size. Matrix corrections employed the PAP algorithm (Pouchou and Pichoir 1985), with oxygen content calculated by stoichiometry. Standard materials were natural crystalline solids for all elements, for which NIST glass K309 was used. Counting times were $30 \mathrm{~s}$ on peak for all elements, yielding minimum levels of detection (calculated at $3 \sigma$ above mean background) in the range of 0.01-0.02 wt. \% of the oxides.

Major elements were analyzed by X-ray fluorescence (XRF) apparatus Rigaku RIX 2000 with a Rh tube hosted at Naruto University in Japan. Glass beads from finely ground samples were prepared with a sampleto-flux $\left(\mathrm{Li}_{2} \mathrm{~B}_{4} \mathrm{O}_{7}\right)$ ratio of 1:10 and the method yielded analytical errors $<1 \%$. Some major- and trace-element compositions, including REE, were determined at ALS Chemex, Vancouver, Canada by ICP-AES and ICP-MS techniques. Major elements were analyzed by ICP-AES (detection limit $\sim 0.01 \%$ ). Trace-element analyses were carried out by ICP-MS (detection limit of 0.01-0.1 ppm), following Li metaborate fusion and $\mathrm{HNO}_{3}$ total digestion. Details of the chemical procedures are found

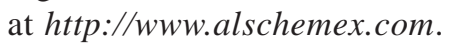

\section{Geological setting}

\subsection{Kuh-e Dom intrusion}

This intrusion is an arc segment of the UrumiehDokhtar magmatic belt. It includes gabbro, diorite, quartz diorite, monzodiorite, monzonite, quartz monzonite, quartz monzodiorite, monzogranite and granodiorite, and - based on $\mathrm{K}$-Ar dating studies - was emplaced in Late Eocene (Technoexport 1981). The intermediate-basic units form a discontinuous band surrounding the northern, southern and eastern margins. The contact zone where I-type, high-K calc-alkaline, metaluminous Kuh-e Dom plutons (Sarjoughian 2007) intruded Paleozoic schists, Cretaceous limestones and early Eocene volcanic rocks (Fig. 1) is marked by a thermal metamorphic aureole with skarn and hornfels. Crosscutting relations show that the intermediate-basic are relatively younger than the silicic igneous rocks. The whole-rock geochemical signature indicates that the parental magma may have been derived from a mantle and lower crust sources carrying a subductionrelated imprint. Underplating of already thickened continental crust by mantle-derived hot basic magma was proposed to have resulted in dehydration melting of the lower crust and mixing between the contrasting magmas (Sarjoughian 2012).

\subsection{Silicic dikes}

The silicic dikes (porphyric microgranites, porphyric microgranodiorites and aplites) are oriented W-E to NWSE, forming c. $10 \%$ of igneous exposures. They tend to be cream or light gray in color. The dikes intruded silicic and intermediate-basic units of the intrusion and the host rocks, especially in its southern and southeastern parts. The dikes generally show subhorizontal orientations, although sometimes they clearly cross cut each other. Although the thicknesses of silicic dikes vary between 0.5 and 20 meters, most are 5 to 6 meters wide. The studied 
(a)

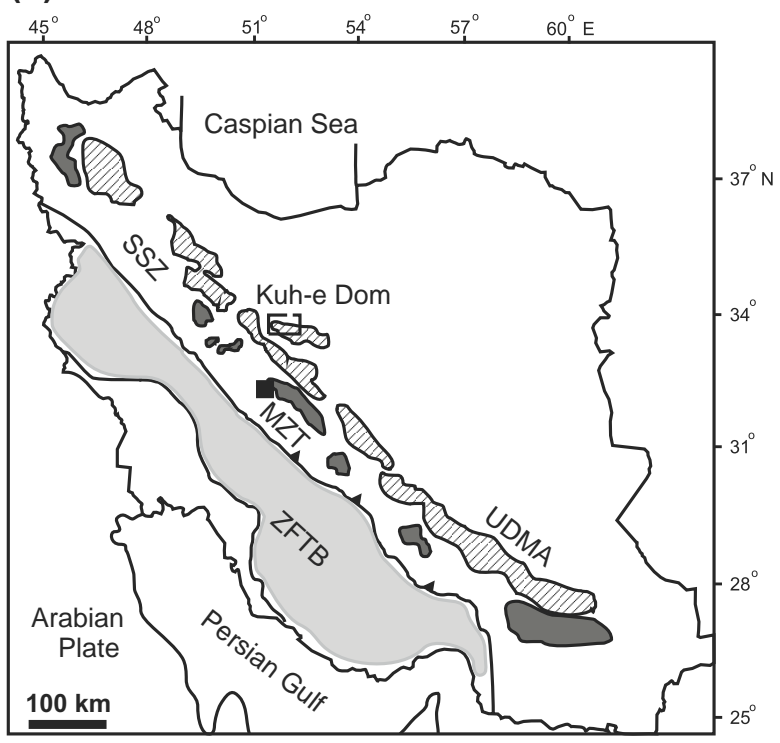

WZFTB: Zagros Fold-Thrust Belt

Sanandaj-Sirjan Basins

UDMA: Urumieh-Dokhtar

Magmatic Arc

(b)

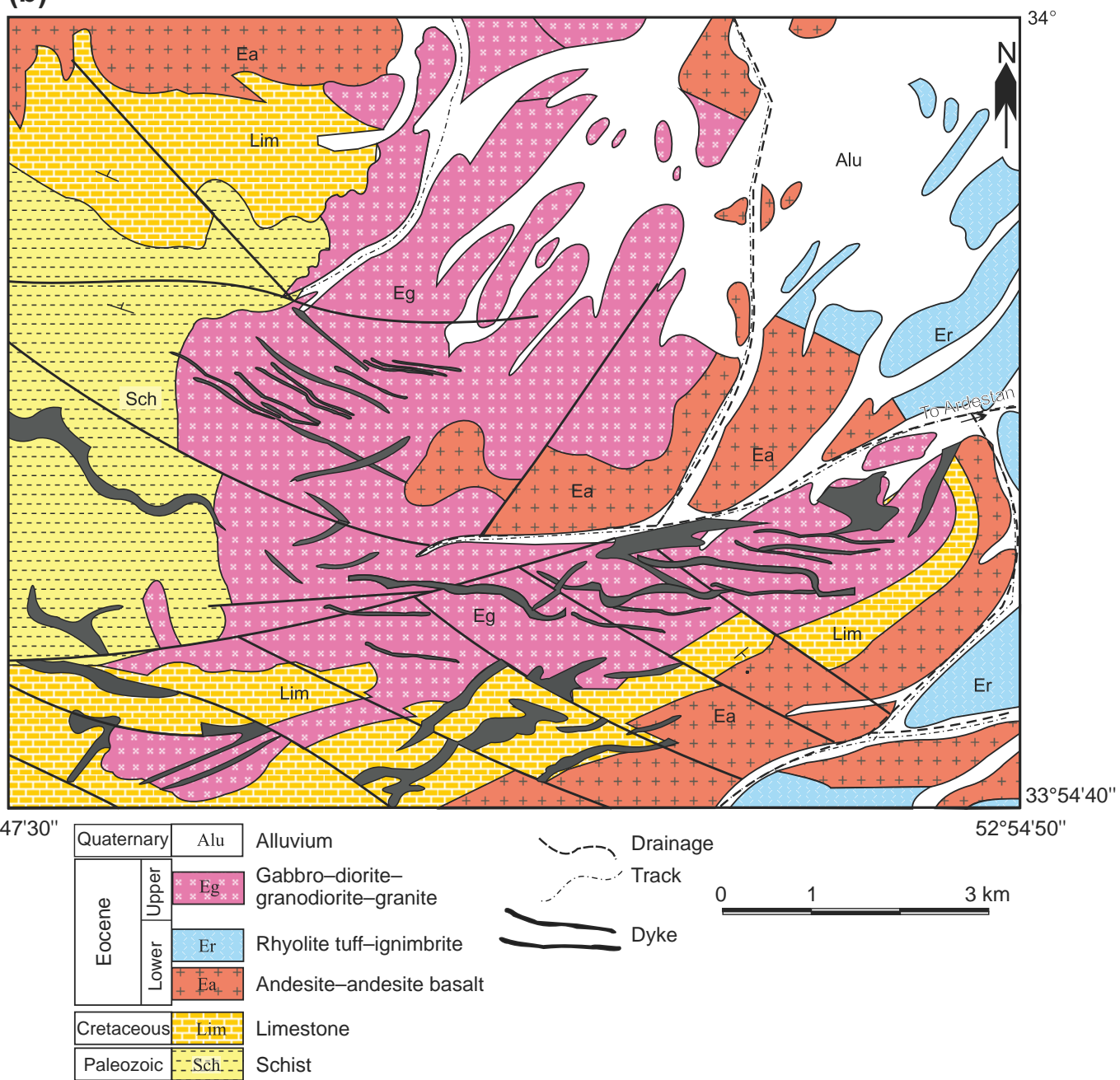

Fig. 1a Generalized tectonic map of Iran: UDMA = Urumieh-Dokhtar Magmatic Arc, SSZ = Sanandaj-Sirjan Zone, MZT = Main Zagros Thrust. b - Simplified geological map of Kuh-e Dom intrusion adopted from Technoexport (1981), slightly modified. 

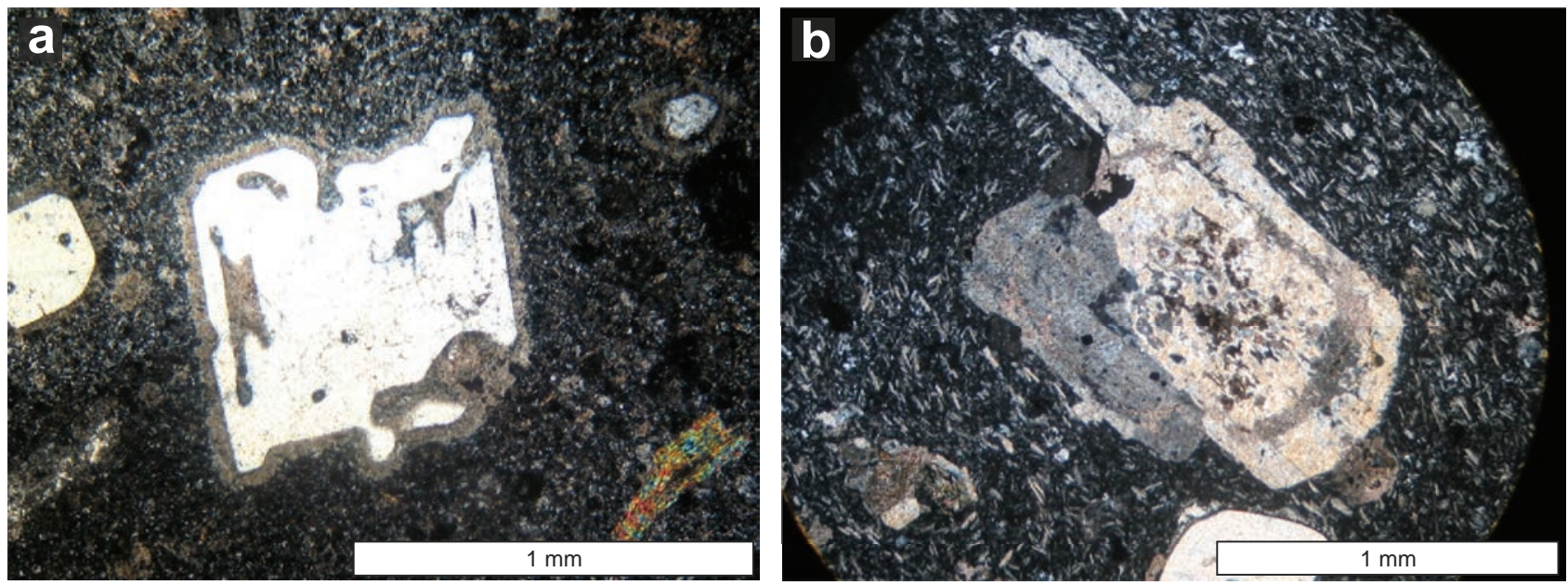

Fig. 2 Photomicrographs (XPL) of porphyritic microgranite (H3) with corroded quartz phenocrysts surrounded by reaction rims (a) and porphyritic microgranodiorite (F12) with sieve texture in plagioclase (b).

dikes are characterized mostly by porphyric texture and minor chloritization. Contacts between the dikes and plutonic host rocks are sharp and planar, implying that the pluton was rigid at the time of dike emplacement. The dikes with chilled margins indicate emplacement at shallow depths after the coarse-grained granite had solidified.

\subsection{Intermediate-basic dikes}

The dark green or brown intermediate-basic dikes cutting the Kuh-e Dom intrusion and host rocks are oriented $\mathrm{W}-\mathrm{E}$ and occur less frequently than their silicic counterpants. They are chemically classified as phonolite, basanite and trachyandesite with partly aphanitic and porphyric texture, fine to medium-grained. These dikes are c. 1 meter thick and have no obvious chilled margins. Most intermediate-basic dikes show hydrothermal alteration with local calcitic, chloritic, epidotic and spilititic veins.

\section{Petrography}

\subsection{Silicic dikes}

The rock-forming minerals include quartz, alkali-feldspar, plagioclase, biotite and rarely hornblende, all of which occur both as phenocrysts and in the groundmass. The phenocrysts are present either as isolated phenocrysts or as glomerocrysts.

Alkali-feldspar and plagioclase usually dominate both the phenocryst assemblage and the groundmass. However, some porphyric microgranite dikes contain more than $90 \%$ of quartz phenocrysts with low contents of alkali-feldspar, plagioclase and biotite phenocrysts. The coarse quartz grains often show corroded margins; some contain dissolution cavities in central parts.
Quartz phenocrysts are surrounded by reaction rims of fine-grained feldspars (Fig. 2a), which indicate disequilibrium of the quartz crystals with the surrounding magma, caused by changes in melt pressure or chemical composition (Vernon 2004).

Alkali-feldspars occur more frequently in porphyric microgranite than in the porphyric microgranodiorite. The latter shows large amounts of plagioclase phenocrysts and or as microlites. The phenocrysts are often euhedral indicating early crystal growth, whereas the plagioclase microlites form the groundmass of these rocks. Some large crystals have dissolved margins caused by reactions with the surrounding magma. Plagioclase phenocrysts show polysynthetic twinning and/or oscillatory zoning indicating a slow equilibration relative to the crystallization rate (Vernon 2004).

Plagioclases enclose various mineral inclusions and show poikilitic and, rarely, sieve-textures (Fig. 2b). The sieve texture is apparently caused by changes in crystal growth conditions causing dissolution of parts of the crystal. As the crystals continued to grow, portions of melt were surrounded by plagioclase (Vernon 2004). The mafic minerals of these dikes are common euhedral, fineto medium-grained biotites and rare euhedral amphiboles. The phenocrysts are embedded in a fine-grained groundmass formed of quartz and feldspar, and some biotite. Apatite and zircon are accessory minerals. Some dikes show sericitization and kaolinization of alkali feldspars, saussuritization and carbonatization of plagioclase, and chloritization of biotites and hornblendes.

The aplites consist of abundant anhedral quartz, alkalifeldspar and rarely albitic plagioclase and biotite. The alkalifeldspars tend to be kaolinized and show perthitic texture. The plagioclases often show polysynthetic twinning and are slightly sericitized. Biotite as anhedral crystals is the only ferromagnesian mineral; it is commonly altered to chlorite. 

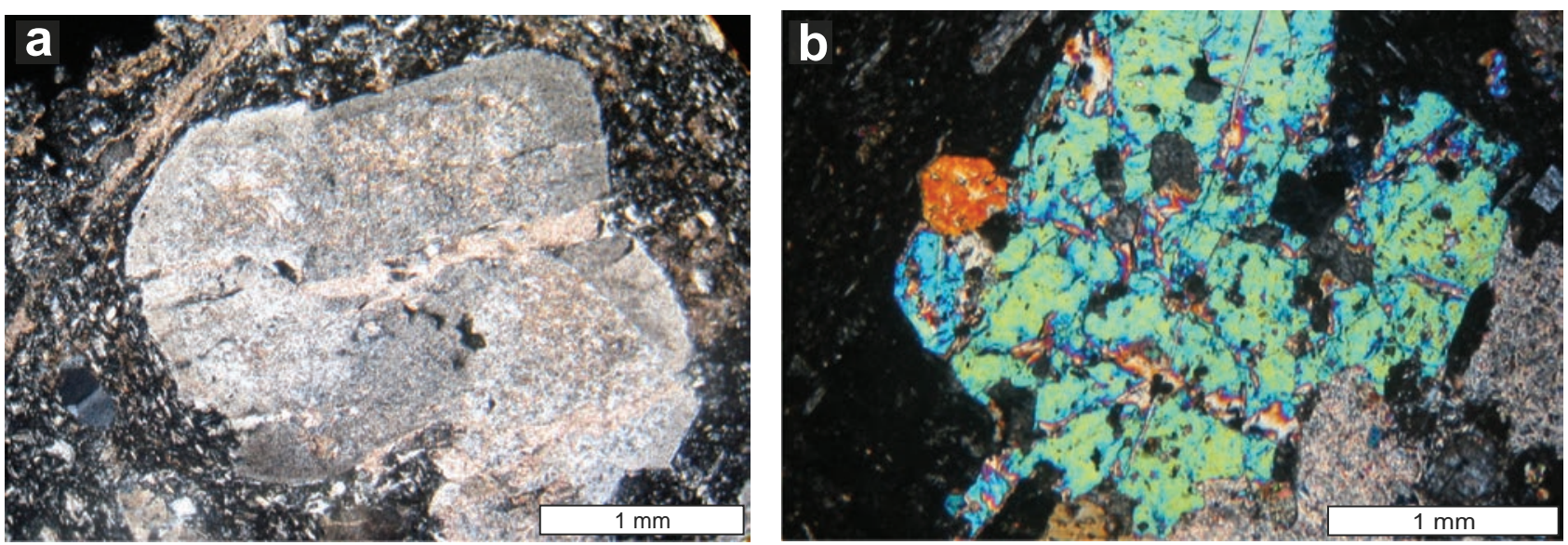

Fig. 3 Photomicrographs (XPL) of intermediate-basic dikes. a - Corroded plagioclase with discontinuous zoning (S10). b - Poikilitic texture of clinopyroxene (S35).

\subsection{Intermediate-basic dikes}

The intermediate-basic dikes usually show porphyric and, sometimes, microlitic textures. They are composed of $60-70 \%$ pyroxene and plagioclase phenocrysts set in groundmass of plagioclase microlites with fine-grained pyroxene, plagioclase, amphibole, biotite and alkali-feldspar. Basanites and trachyandesite are fine-to medium-grained and differ from phonolites by the presence of abundant pyroxene, lower feldspars and higher mafic minerals contents. Plagioclase is the dominant phenocryst phase, forming mainly subhedral and lath-shaped crystals ( $0.3 \mathrm{~mm}$ to several $\mathrm{mm}$ across). Frequently they show corroded margins (Fig. 3a) and enclose many inclusions of clinopyroxene and opaque minerals. The plagioclase crystals show polysynthetic twinning, oscillatory and/or discontinuous zoning, as well as sieve textures. Some grains are altered to sericite and clay minerals.

Subhedral clinopyroxene is the main mafic phase in the dikes. This mineral encloses plagioclase and mafic mineral inclusions, forming poikilitic textures (Fig. 3b). It suffered intense alteration; some clinopyroxenes are partially replaced by actinolitic amphibole, others are completely uralitized and carbonatized.

Rare euhedral prismatic amphibole crystals of green color are associated with biotite and slightly altered to chlorite. Biotite is generally subhedral, pleochroic from reddish brown to brown and often altered to titanite and chlorite. K-feldspar mostly occupies the interstices between plagioclase laths and other minerals, suggesting late crystallization. Some of the K-feldspar rims the plagioclase phenocrysts. Acicular apatite, sphene and opaque minerals are common accessory phases. Some of the dikes were altered to spilite and are composed now of oligoclase, together with chlorite, epidote, calcite, and actinolite.

\section{Chemical composition of minerals}

The results of electron-microprobe analysis are shown in Tables 1-5. Mineral recalculations were done by the MinPet Geological Software version 2.02 (Richard 1995). Structural formulae of feldspars were calculated on the basis of 32 oxygens. Pyroxenes were classified by using the total numbers of specified cations at the $\mathrm{M}$ sites on the basis of $6 \mathrm{O}$ atoms. The $\mathrm{Fe}^{3+}$ estimates were made using the general equation of Droop (1987) assuming 4 cations per formula unit. Amphibole analyses were recalculated on anhydrous basis to cations per 23 oxygens. Ferrous and ferric iron were assessed by the charge balance method described by Robinson et al. (1981), using the average between the 15-NK and 13-CNK methods after applying the appropriate IMA site distribution rules (Leake et al. 1997). Biotite formulae were recalculated based on $24 \mathrm{O}$ atoms and no $\mathrm{OH}$ groups; the oxidation state of iron was assessed by the $\mathrm{Mica}^{+}$software (Yavuz 2003).

In the ternary Ab-Or-An diagram (Deer et al. 1992), plagioclase compositions in the silicic dikes are albite $\left(\mathrm{An}_{3-9}\right)$ and in the intermediate-basic dikes andesine, labradorite, and anorthite $\left(\mathrm{An}_{30-56}\right.$ and $\mathrm{An}_{98}$; Fig. 4). Alkali-feldspar in intermediate-basic dikes corresponds to orthoclase with Or content between 60 and 95 \% (Fig. 4).

In the intermediate dikes, biotites with high $\mathrm{TiO}_{2}$ contents are primary (Nachit et al. 2005) and classify (Rieder et al. 1998) as biotite close to phlogopite end member $\left(\mathrm{X}_{\mathrm{Mg}}=\mathrm{Mg} /(\mathrm{Mg}+\mathrm{Fe}) \sim 0.63\right.$; Fig. 5a). The alumina saturation index (ASI) of biotites $\left[\mathrm{Al}^{\mathrm{T}} /(\mathrm{Ca}+\mathrm{Na}+\mathrm{K})\right]$ is low (1.28) and reflects lower alumina activity in the magma (Zen 1988). Based on $\mathrm{FeO}$ and $\mathrm{Al}_{2} \mathrm{O}_{3}$ contents, all analyses plot into the calc-alkaline field (Fig. 5b; AbdelRahman 1994). Mg-rich micas with low $\mathrm{Fe}^{2+} /\left(\mathrm{Mg}+\mathrm{Fe}^{2+}\right)$ ratios plot in the shoshonitic field (Fig. 5c; Jiang et al. 2002), indicative of high $\mathrm{fO}_{2}$ conditions of the magmas during crystallization. 
Tab. 1 Electron-microprobe analyses of plagioclase in Kuh-e Dom dikes (wt. \% and apfu calculated on the basis of 32 O).

\begin{tabular}{|c|c|c|c|c|c|c|c|c|}
\hline Sample & F117-21 & F117-22 & F117-41 & F117-42 & F117-43 & F117-44 & S28-1 & S28-3 \\
\hline Location & $\operatorname{dik}$ A & $\operatorname{dik}$ A & $\operatorname{dik}$ A & $\operatorname{dik}$ A & $\operatorname{dik} \mathrm{A}$ & $\operatorname{dik}$ A & $\operatorname{dik} B$ & dik B \\
\hline $\mathrm{SiO}_{2}$ & 67.55 & 68.33 & 66.83 & 68.98 & 64.32 & 68.15 & 53.91 & 56.00 \\
\hline $\mathrm{TiO}_{2}$ & 0.00 & 0.01 & 0.00 & 0.00 & 0.00 & 0.03 & 0.03 & 0.16 \\
\hline $\mathrm{Al}_{2} \mathrm{O}_{3}$ & 20.03 & 19.65 & 20.02 & 19.51 & 20.75 & 19.91 & 28.84 & 27.44 \\
\hline $\mathrm{FeO}$ & 0.12 & 0.09 & 0.55 & 0.05 & 0.49 & 0.05 & 0.58 & 0.50 \\
\hline $\mathrm{MnO}$ & 0.01 & 0.00 & 0.00 & 0.01 & 0.00 & 0.00 & 0.00 & 0.01 \\
\hline MgO & 0.04 & 0.02 & 0.09 & 0.00 & 0.12 & 0.01 & 0.04 & 0.05 \\
\hline $\mathrm{BaO}$ & 0.09 & 0.11 & 0.04 & 0.09 & 0.10 & 0.18 & 0.05 & 0.04 \\
\hline $\mathrm{CaO}$ & 0.12 & 0.12 & 0.06 & 0.06 & 0.09 & 0.19 & 10.94 & 9.43 \\
\hline $\mathrm{Na}_{2} \mathrm{O}$ & 11.55 & 11.47 & 11.36 & 11.76 & 10.24 & 11.83 & 5.19 & 6.06 \\
\hline $\mathrm{K}_{2} \mathrm{O}$ & 0.27 & 0.20 & 0.46 & 0.12 & 1.63 & 0.11 & 0.24 & 0.21 \\
\hline $\mathrm{SrO}$ & 0.02 & 0.03 & 0.03 & 0.01 & 0.01 & $*$ & 0.15 & 0.10 \\
\hline Total & 99.80 & 100.03 & 99.44 & 100.59 & 97.75 & 100.46 & 99.97 & 100.00 \\
\hline $\mathrm{Si}$ & 11.86 & 11.95 & 11.81 & 11.99 & 11.63 & 11.89 & 9.78 & 10.10 \\
\hline $\mathrm{Al}$ & 4.14 & 4.05 & 4.17 & 3.99 & 4.42 & 4.09 & 6.16 & 5.83 \\
\hline $\mathrm{Ti}$ & 0.00 & 0.00 & 0.00 & 0.00 & 0.00 & 0.00 & 0.00 & 0.02 \\
\hline $\mathrm{Fe}^{2+}$ & 0.02 & 0.01 & 0.08 & 0.01 & 0.07 & 0.01 & 0.09 & 0.08 \\
\hline Mn & 0.00 & 0.00 & 0.00 & 0.00 & 0.00 & 0.00 & 0.00 & 0.00 \\
\hline Mg & 0.01 & 0.01 & 0.02 & 0.00 & 0.03 & 0.00 & 0.01 & 0.01 \\
\hline $\mathrm{Ba}$ & 0.01 & 0.01 & 0.00 & 0.01 & 0.01 & 0.01 & 0.00 & 0.00 \\
\hline $\mathrm{Ca}$ & 0.02 & 0.02 & 0.01 & 0.01 & 0.02 & 0.04 & 2.13 & 1.82 \\
\hline $\mathrm{Na}$ & 3.93 & 3.89 & 3.89 & 3.96 & 3.59 & 4.00 & 1.83 & 2.12 \\
\hline $\mathrm{K}$ & 0.06 & 0.05 & 0.10 & 0.03 & 0.38 & 0.02 & 0.06 & 0.05 \\
\hline $\mathrm{Ab}$ & 97.90 & 98.30 & 97.10 & 99.10 & 90.10 & 98.50 & 45.50 & 53.10 \\
\hline An & 0.60 & 0.60 & 0.30 & 0.30 & 0.40 & 0.90 & 53.10 & 45.70 \\
\hline Or & 1.50 & 1.10 & 2.60 & 0.70 & 9.40 & 0.60 & 1.40 & 1.20 \\
\hline Sample & S28-14 & S28-15 & S282 & S28-21 & S28-22 & S28-3 & S28-31 & S28-32 \\
\hline Location & $\operatorname{dik} B$ & dik B & dik B & dik B & dik B & $\operatorname{dik} B$ & dik B & dik B \\
\hline $\mathrm{SiO}_{2}$ & 53.40 & 58.49 & 53.77 & 54.01 & 53.95 & 53.11 & 52.76 & 54.15 \\
\hline $\mathrm{TiO}_{2}$ & 0.07 & 0.03 & 0.07 & 0.08 & 0.09 & 0.11 & 0.06 & 0.06 \\
\hline $\mathrm{Al}_{2} \mathrm{O}_{3}$ & 29.65 & 25.37 & 28.95 & 28.58 & 28.71 & 29.00 & 28.79 & 28.70 \\
\hline $\mathrm{FeO}$ & 0.43 & 0.39 & 0.43 & 0.44 & 0.54 & 0.70 & 1.76 & 1.20 \\
\hline $\mathrm{MnO}$ & 0.00 & 0.01 & 0.01 & 0.01 & 0.03 & 0.02 & 0.00 & 0.01 \\
\hline MgO & 0.00 & 0.13 & 0.09 & 0.07 & 0.10 & 0.03 & 0.07 & 0.06 \\
\hline $\mathrm{BaO}$ & 0.09 & 0.07 & 0.11 & 0.10 & 0.05 & 0.04 & 0.11 & 0.09 \\
\hline $\mathrm{CaO}$ & 11.82 & 6.84 & 11.06 & 10.91 & 10.99 & 11.44 & 11.38 & 10.79 \\
\hline $\mathrm{Na}_{2} \mathrm{O}$ & 4.84 & 7.50 & 5.06 & 5.17 & 5.20 & 4.85 & 4.89 & 5.26 \\
\hline $\mathrm{K}_{2} \mathrm{O}$ & 0.17 & 0.87 & 0.24 & 0.25 & 0.25 & 0.17 & 0.21 & 0.19 \\
\hline $\mathrm{SrO}$ & 0.15 & 0.11 & 0.15 & 0.18 & 0.16 & 0.15 & 0.18 & 0.15 \\
\hline Total & 100.62 & 99.81 & 99.94 & 99.80 & 100.07 & 99.62 & 100.21 & 100.66 \\
\hline $\mathrm{Si}$ & 9.64 & 10.54 & 9.76 & 9.82 & 9.79 & 9.69 & 9.64 & 9.79 \\
\hline $\mathrm{Al}$ & 6.31 & 5.38 & 6.19 & 6.12 & 6.13 & 6.23 & 6.19 & 6.11 \\
\hline $\mathrm{Ti}$ & 0.01 & 0.00 & 0.01 & 0.01 & 0.01 & 0.02 & 0.01 & 0.01 \\
\hline $\mathrm{Fe}^{2+}$ & 0.07 & 0.06 & 0.07 & 0.07 & 0.08 & 0.11 & 0.27 & 0.18 \\
\hline Mn & 0.00 & 0.00 & 0.00 & 0.00 & 0.01 & 0.00 & 0.00 & 0.00 \\
\hline $\mathrm{Mg}$ & 0.00 & 0.04 & 0.02 & 0.02 & 0.03 & 0.01 & 0.02 & 0.02 \\
\hline $\mathrm{Ba}$ & 0.01 & 0.01 & 0.01 & 0.01 & 0.00 & 0.00 & 0.01 & 0.01 \\
\hline $\mathrm{Ca}$ & 2.29 & 1.32 & 2.15 & 2.13 & 2.14 & 2.24 & 2.23 & 2.09 \\
\hline $\mathrm{Na}$ & 1.70 & 2.62 & 1.78 & 1.82 & 1.83 & 1.72 & 1.73 & 1.84 \\
\hline $\mathrm{K}$ & 0.04 & 0.20 & 0.06 & 0.06 & 0.06 & 0.04 & 0.05 & 0.04 \\
\hline $\mathrm{Ab}$ & 42.20 & 63.30 & 44.70 & 45.50 & 45.50 & 43.00 & 43.20 & 46.40 \\
\hline An & 56.90 & 31.90 & 53.90 & 53.10 & 53.10 & 56.00 & 55.60 & 52.50 \\
\hline Or & 1.00 & 4.80 & 1.40 & 1.40 & 1.40 & 1.00 & 1.20 & 1.10 \\
\hline
\end{tabular}




\begin{tabular}{|c|c|c|c|c|c|c|c|c|}
\hline Sample & S28-33 & S28-34 & S28-35 & S28-4 & S28-5 & S28-6 & S35-51 & S35-52 \\
\hline Location & $\operatorname{dik} B$ & dik B & dik B & dik B & $\operatorname{dik} B$ & $\operatorname{dik} B$ & dik B & dik B \\
\hline $\mathrm{SiO}_{2}$ & 53.06 & 54.46 & 53.40 & 54.78 & 53.13 & 60.03 & 46.16 & 45.93 \\
\hline $\mathrm{TiO}_{2}$ & 0.08 & 0.06 & 0.09 & 0.09 & 0.08 & 0.04 & 0.02 & 0.02 \\
\hline $\mathrm{Al}_{2} \mathrm{O}_{3}$ & 28.65 & 28.20 & 29.14 & 28.17 & 29.09 & 24.77 & 24.91 & 24.96 \\
\hline $\mathrm{FeO}$ & 1.09 & 0.29 & 0.42 & 0.63 & 0.42 & 0.32 & 0.82 & 0.63 \\
\hline $\mathrm{MnO}$ & 0.00 & 0.00 & 0.01 & 0.01 & 0.00 & 0.00 & 0.02 & 0.04 \\
\hline $\mathrm{MgO}$ & 0.18 & 0.01 & 0.06 & 0.09 & 0.12 & 0.04 & 0.03 & 0.00 \\
\hline $\mathrm{BaO}$ & 0.08 & 0.11 & 0.12 & 0.10 & 0.04 & 0.07 & 27.66 & 27.13 \\
\hline $\mathrm{CaO}$ & 11.34 & 10.64 & 11.49 & 10.47 & 11.46 & 6.34 & 0.06 & 0.06 \\
\hline $\mathrm{Na}_{2} \mathrm{O}$ & 4.83 & 5.35 & 4.87 & 5.51 & 4.81 & 7.86 & 0.09 & 0.24 \\
\hline $\mathrm{K}_{2} \mathrm{O}$ & 0.19 & 0.22 & 0.25 & 0.27 & 0.22 & 0.32 & 0.07 & 0.09 \\
\hline $\mathrm{SrO}$ & 0.14 & 0.16 & 0.17 & 0.16 & 0.19 & 0.09 & 0.07 & 0.34 \\
\hline Total & 99.64 & 99.50 & 100.02 & 100.28 & 99.56 & 99.88 & 99.91 & 99.44 \\
\hline $\mathrm{Al}$ & 6.17 & 6.04 & 6.23 & 6.00 & 6.25 & 5.22 & 5.62 & 5.66 \\
\hline $\mathrm{Ti}$ & 0.01 & 0.01 & 0.01 & 0.01 & 0.01 & 0.01 & 0.00 & 0.00 \\
\hline $\mathrm{Fe}^{2+}$ & 0.17 & 0.04 & 0.06 & 0.10 & 0.06 & 0.05 & 0.13 & 0.10 \\
\hline $\mathrm{Mn}$ & 0.00 & 0.00 & 0.00 & 0.00 & 0.00 & 0.00 & 0.00 & 0.01 \\
\hline $\mathrm{Mg}$ & 0.05 & 0.00 & 0.02 & 0.02 & 0.03 & 0.01 & 0.01 & 0.00 \\
\hline $\mathrm{Ba}$ & 0.01 & 0.01 & 0.01 & 0.01 & 0.00 & 0.01 & 0.01 & 0.02 \\
\hline $\mathrm{Ca}$ & 2.22 & 2.07 & 2.24 & 2.03 & 2.24 & 1.22 & 5.68 & 5.60 \\
\hline $\mathrm{Na}$ & 1.71 & 1.89 & 1.72 & 1.93 & 1.70 & 2.73 & 0.03 & 0.04 \\
\hline $\mathrm{K}$ & 0.04 & 0.05 & 0.06 & 0.06 & 0.05 & 0.07 & 0.02 & 0.08 \\
\hline $\mathrm{Ab}$ & 43.00 & 47.00 & 42.80 & 48.00 & 42.60 & 67.90 & 0.50 & 0.60 \\
\hline An & 55.80 & 51.70 & 55.80 & 50.40 & 56.10 & 30.30 & 99.20 & 97.90 \\
\hline Or & 1.10 & 1.30 & 1.40 & 1.50 & 1.30 & 1.80 & 0.30 & 1.50 \\
\hline
\end{tabular}

\begin{tabular}{|c|c|c|c|c|c|c|c|}
\hline $\begin{array}{l}\text { Sample } \\
\text { Location }\end{array}$ & $\begin{array}{l}\text { S35-53 } \\
\text { dik B }\end{array}$ & $\begin{array}{l}\text { S35-51 } \\
\text { dik B }\end{array}$ & $\begin{array}{l}\text { S35-61 } \\
\text { dik B }\end{array}$ & $\begin{array}{l}\text { S35-62 } \\
\text { dik B }\end{array}$ & $\begin{array}{l}\text { S35-63 } \\
\text { dik B }\end{array}$ & $\begin{array}{l}\text { S35-64 } \\
\text { dik B }\end{array}$ & $\begin{array}{l}\text { S35-65 } \\
\text { dik B }\end{array}$ \\
\hline $\mathrm{SiO}_{2}$ & 46.02 & 46.28 & 45.95 & 45.76 & 45.68 & 46.19 & 45.39 \\
\hline $\mathrm{TiO}_{2}$ & 0.03 & 0.01 & 0.01 & 0.13 & 0.04 & 0.01 & 0.08 \\
\hline $\mathrm{Al}_{2} \mathrm{O}_{3}$ & 24.91 & 24.62 & 24.87 & 24.91 & 25.03 & 25.16 & 24.68 \\
\hline $\mathrm{FeO}$ & 0.65 & 1.28 & 0.93 & 0.80 & 1.31 & 0.59 & 0.78 \\
\hline $\mathrm{MnO}$ & 0.02 & 0.04 & 0.04 & 0.02 & 0.03 & 0.04 & 0.05 \\
\hline $\mathrm{MgO}$ & 0.00 & 0.00 & 0.02 & 0.03 & 0.18 & 0.00 & 0.06 \\
\hline $\mathrm{BaO}$ & 27.40 & 26.83 & 27.15 & 27.77 & 27.21 & 27.60 & 27.68 \\
\hline $\mathrm{CaO}$ & 0.09 & 0.05 & 0.01 & 0.01 & 0.05 & 0.00 & 0.02 \\
\hline $\mathrm{Na}_{2} \mathrm{O}$ & 0.10 & 0.17 & 0.11 & 0.00 & 0.10 & 0.09 & 0.15 \\
\hline $\mathrm{K}_{2} \mathrm{O}$ & 0.12 & 0.07 & 0.09 & 0.06 & 0.05 & 0.06 & 0.17 \\
\hline $\mathrm{SrO}$ & 0.13 & 0.40 & 0.28 & 0.06 & 0.05 & 0.13 & 0.16 \\
\hline Total & 99.49 & 99.73 & 99.47 & 99.55 & 99.74 & 99.87 & 99.21 \\
\hline $\mathrm{Al}$ & 5.65 & 5.58 & 5.64 & 5.64 & 5.67 & 5.67 & 5.63 \\
\hline $\mathrm{Ti}$ & 0.01 & 0.00 & 0.00 & 0.02 & 0.01 & 0.00 & 0.01 \\
\hline $\mathrm{Fe}^{2+}$ & 0.11 & 0.21 & 0.15 & 0.13 & 0.21 & 0.09 & 0.13 \\
\hline $\mathrm{Mn}$ & 0.00 & 0.01 & 0.01 & 0.00 & 0.01 & 0.01 & 0.01 \\
\hline $\mathrm{Mg}$ & 0.00 & 0.00 & 0.01 & 0.01 & 0.05 & 0.00 & 0.02 \\
\hline $\mathrm{Ba}$ & 0.01 & 0.01 & 0.01 & 0.00 & 0.01 & 0.01 & 0.01 \\
\hline $\mathrm{Ca}$ & 5.65 & 5.53 & 5.60 & 5.72 & 5.61 & 5.66 & 5.74 \\
\hline $\mathrm{Na}$ & 0.04 & 0.02 & 0.04 & 0.02 & 0.02 & 0.02 & 0.06 \\
\hline $\mathrm{K}$ & 0.03 & 0.10 & 0.07 & 0.02 & 0.01 & 0.03 & 0.04 \\
\hline $\mathrm{Ab}$ & 0.80 & 0.40 & 0.60 & 0.40 & 0.40 & 0.40 & 1.10 \\
\hline An & 98.70 & 97.90 & 98.20 & 99.30 & 99.40 & 99.10 & 98.30 \\
\hline Or & 0.50 & 1.70 & 1.20 & 0.30 & 0.20 & 0.50 & 0.70 \\
\hline
\end{tabular}

dik A: acidic dikes, dik B: basic-intermediate dikes 
Tab. 2 Electron-microprobe analyses of K-feldspar from intermediate-basic dikes (wt. \% and apfu calculated on the basis of 32 O).

\begin{tabular}{|c|c|c|c|c|c|c|c|c|}
\hline Sample & S28-11 & S28-12 & S28-42 & S28-43 & S35-11 & S35-12 & S35-13 & S35-14 \\
\hline $\mathrm{SiO}_{2}$ & 64.11 & 62.87 & 63.09 & 62.26 & 64.07 & 63.23 & 64.08 & 62.46 \\
\hline $\mathrm{TiO}_{2}$ & 0.05 & 0.06 & 0.04 & 0.38 & 0.00 & 0.06 & 0.00 & 0.00 \\
\hline $\mathrm{Al}_{2} \mathrm{O}_{3}$ & 18.52 & 18.06 & 19.43 & 16.28 & 18.50 & 18.58 & 18.63 & 18.32 \\
\hline $\mathrm{FeO}$ & 0.22 & 0.16 & 0.34 & 2.84 & 0.15 & 0.70 & 0.17 & 1.63 \\
\hline $\mathrm{MnO}$ & 0.00 & 0.02 & 0.01 & 0.06 & 0.00 & 0.00 & 0.01 & 0.01 \\
\hline MgO & 0.01 & 0.03 & 0.05 & 2.23 & 0.03 & 0.44 & 0.06 & 1.10 \\
\hline $\mathrm{BaO}$ & 0.44 & 0.43 & 0.41 & 0.42 & 0.61 & 0.62 & 0.33 & 0.67 \\
\hline $\mathrm{CaO}$ & 0.10 & 0.08 & 1.30 & 2.45 & 0.09 & 0.42 & 0.05 & 0.18 \\
\hline $\mathrm{Na}_{2} \mathrm{O}$ & 1.08 & 0.97 & 4.01 & 1.02 & 0.48 & 0.54 & 0.36 & 0.52 \\
\hline $\mathrm{K}_{2} \mathrm{O}$ & 14.68 & 14.99 & 11.17 & 11.78 & 15.91 & 14.87 & 16.01 & 14.39 \\
\hline $\mathrm{SrO}$ & 0.23 & 0.17 & 0.19 & 0.20 & 0.16 & 0.15 & 0.21 & 0.09 \\
\hline Total & 99.44 & 97.84 & 100.04 & 99.92 & 100.00 & 99.61 & 99.91 & 99.37 \\
\hline Si & 11.93 & 11.92 & 11.63 & 11.66 & 11.92 & 11.81 & 11.92 & 11.73 \\
\hline $\mathrm{Al}$ & 4.06 & 4.03 & 4.22 & 3.59 & 4.05 & 4.09 & 4.08 & 4.05 \\
\hline $\mathrm{Ti}$ & 0.01 & 0.01 & 0.01 & 0.05 & 0.00 & 0.01 & 0.00 & 0.00 \\
\hline $\mathrm{Fe}^{2+}$ & 0.03 & 0.03 & 0.05 & 0.45 & 0.02 & 0.11 & 0.03 & 0.26 \\
\hline Mn & 0.00 & 0.00 & 0.00 & 0.01 & 0.00 & 0.00 & 0.00 & 0.00 \\
\hline $\mathrm{Mg}$ & 0.00 & 0.01 & 0.01 & 0.62 & 0.01 & 0.12 & 0.02 & 0.31 \\
\hline $\mathrm{Ba}$ & 0.03 & 0.03 & 0.03 & 0.03 & 0.04 & 0.05 & 0.02 & 0.05 \\
\hline $\mathrm{Ca}$ & 0.02 & 0.02 & 0.26 & 0.49 & 0.02 & 0.08 & 0.01 & 0.04 \\
\hline $\mathrm{Na}$ & 0.39 & 0.36 & 1.43 & 0.37 & 0.17 & 0.20 & 0.13 & 0.19 \\
\hline $\mathrm{K}$ & 3.49 & 3.63 & 2.63 & 2.81 & 3.78 & 3.54 & 3.80 & 3.45 \\
\hline $\mathrm{Ab}$ & 10.00 & 8.90 & 33.20 & 10.10 & 4.40 & 5.10 & 3.30 & 5.10 \\
\hline An & 0.50 & 0.40 & 6.00 & 13.40 & 0.50 & 2.20 & 0.30 & 1.00 \\
\hline Or & 89.50 & 90.70 & 60.80 & 76.60 & 95.20 & 92.70 & 96.40 & 93.90 \\
\hline Sample & S35-15 & S35-16 & & S35-61 & S35-62 & S35-63 & S35-71 & S35-72 \\
\hline $\mathrm{SiO}_{2}$ & 64.53 & 61.83 & & 63.40 & 62.59 & 63.12 & 64.22 & 64.30 \\
\hline $\mathrm{TiO}_{2}$ & 0.03 & 0.00 & & 0.00 & 0.07 & 0.00 & 0.01 & 0.03 \\
\hline $\mathrm{Al}_{2} \mathrm{O}_{3}$ & 18.09 & 18.24 & & 17.99 & 19.01 & 18.86 & 18.81 & 18.75 \\
\hline $\mathrm{FeO}$ & 0.08 & 2.06 & & 0.41 & 1.33 & 0.27 & 0.02 & 0.02 \\
\hline $\mathrm{MnO}$ & 0.00 & 0.03 & & 0.01 & 0.02 & 0.01 & 0.00 & 0.01 \\
\hline MgO & 0.03 & 1.27 & & 0.32 & 0.44 & 0.07 & 0.00 & 0.01 \\
\hline $\mathrm{BaO}$ & 0.26 & 0.57 & & 0.67 & 0.85 & 0.66 & 0.35 & 0.32 \\
\hline $\mathrm{CaO}$ & 0.05 & 0.22 & & 0.17 & 0.15 & 0.24 & 0.00 & 0.01 \\
\hline $\mathrm{Na}_{2} \mathrm{O}$ & 0.35 & 0.36 & & 0.44 & 0.41 & 0.41 & 0.41 & 0.39 \\
\hline $\mathrm{K}_{2} \mathrm{O}$ & 16.03 & 14.37 & & 15.29 & 14.98 & 15.66 & 15.83 & 15.92 \\
\hline $\mathrm{SrO}$ & 0.13 & 0.15 & & 0.22 & 0.22 & 0.19 & 0.55 & 0.46 \\
\hline Total & 99.58 & 99.10 & & 98.92 & 100.07 & 99.49 & 100.20 & 100.22 \\
\hline Si & 12.01 & 11.67 & & 11.93 & 11.70 & 11.82 & 11.92 & 11.93 \\
\hline Al & 3.97 & 4.06 & & 3.99 & 4.19 & 4.16 & 4.11 & 4.10 \\
\hline $\mathrm{Ti}$ & 0.00 & 0.00 & & 0.00 & 0.01 & 0.00 & 0.00 & 0.00 \\
\hline $\mathrm{Fe}^{2+}$ & 0.01 & 0.33 & & 0.07 & 0.21 & 0.04 & 0.00 & 0.00 \\
\hline $\mathrm{Mn}$ & 0.00 & 0.01 & & 0.00 & 0.00 & 0.00 & 0.00 & 0.00 \\
\hline $\mathrm{Mg}$ & 0.01 & 0.36 & & 0.09 & 0.12 & 0.02 & 0.00 & 0.00 \\
\hline $\mathrm{Ba}$ & 0.02 & 0.04 & & 0.05 & 0.06 & 0.05 & 0.03 & 0.02 \\
\hline $\mathrm{Ca}$ & 0.01 & 0.04 & & 0.03 & 0.03 & 0.05 & 0.00 & 0.00 \\
\hline $\mathrm{Na}$ & 0.13 & 0.13 & & 0.16 & 0.15 & 0.15 & 0.15 & 0.14 \\
\hline $\mathrm{K}$ & 3.81 & 3.46 & & 3.67 & 3.57 & 3.74 & 3.75 & 3.77 \\
\hline $\mathrm{Ab}$ & 3.20 & 3.60 & & 4.20 & 4.00 & 3.80 & 3.80 & 3.60 \\
\hline An & 0.30 & 1.20 & & 0.90 & 0.80 & 1.20 & 0.00 & 0.10 \\
\hline Or & 96.50 & 95.20 & & 95.00 & 95.20 & 95.00 & 96.20 & 96.40 \\
\hline
\end{tabular}


Tab. 3 Electron-microprobe analyses of biotite from intermediate dikes (wt. \% and apfu recalculated based on 24 O).

\begin{tabular}{|c|c|c|c|c|c|c|c|c|}
\hline Sample & S28-11 & S28.2 & S28-13 & S28-31 & S28-32 & S28-41 & S28-.2 & S28-43 \\
\hline $\mathrm{SiO}_{2}$ & 37.67 & 37.58 & 37.48 & 37.86 & 38.07 & 38.25 & 37.99 & 37.94 \\
\hline $\mathrm{TiO}_{2}$ & 4.37 & 4.26 & 4.58 & 4.55 & 4.41 & 4.37 & 4.31 & 4.03 \\
\hline $\mathrm{Al}_{2} \mathrm{O}_{3}$ & 13.26 & 13.24 & 13.29 & 13.37 & 13.28 & 13.43 & 13.36 & 13.09 \\
\hline $\mathrm{Cr}_{2} \mathrm{O}_{3}$ & 0.02 & 0.01 & 0.02 & 0.03 & 0.03 & 0.03 & 0.04 & 0.04 \\
\hline $\mathrm{FeO}$ & 15.39 & 16.20 & 15.30 & 15.66 & 15.11 & 15.61 & 15.76 & 15.69 \\
\hline $\mathrm{MnO}$ & 0.17 & 0.19 & 0.16 & 0.19 & 0.20 & 0.16 & 0.17 & 0.19 \\
\hline $\mathrm{MgO}$ & 14.58 & 14.59 & 14.46 & 14.92 & 14.64 & 15.02 & 15.14 & 15.02 \\
\hline $\mathrm{BaO}$ & 0.13 & 0.15 & 0.13 & 0.15 & 0.14 & 0.14 & 0.20 & 0.12 \\
\hline $\mathrm{CaO}$ & 0.02 & 0.02 & 0.04 & 0.03 & 0.21 & 0.02 & 0.04 & 0.18 \\
\hline $\mathrm{Na}_{2} \mathrm{O}$ & 0.07 & 0.04 & 0.05 & 0.10 & 0.07 & 0.11 & 0.08 & 0.02 \\
\hline $\mathrm{K}_{2} \mathrm{O}$ & 9.67 & 9.54 & 9.43 & 9.45 & 9.43 & 9.43 & 9.58 & 9.57 \\
\hline F & 0.24 & 0.29 & 0.23 & 0.14 & 0.29 & 0.19 & 0.50 & 0.24 \\
\hline $\mathrm{Cl}$ & 0.32 & 0.30 & 0.28 & 0.31 & 0.29 & 0.29 & 0.29 & 0.32 \\
\hline Total & 95.91 & 96.41 & 95.45 & 96.76 & 96.17 & 97.05 & 97.46 & 96.45 \\
\hline$\overline{\mathrm{Si}}$ & 5.91 & 5.89 & 5.90 & 5.88 & 5.94 & 5.92 & 5.89 & 5.93 \\
\hline $\mathrm{Al}^{\mathrm{IV}}$ & 2.09 & 2.11 & 2.10 & 2.12 & 2.06 & 2.08 & 2.11 & 2.07 \\
\hline $\mathrm{Al}^{\mathrm{VI}}$ & 0.37 & 0.34 & 0.37 & 0.33 & 0.38 & 0.36 & 0.33 & 0.34 \\
\hline $\mathrm{Ti}$ & 0.52 & 0.50 & 0.54 & 0.53 & 0.52 & 0.51 & 0.50 & 0.47 \\
\hline $\mathrm{Fe}^{3+}$ & 0.65 & 0.78 & 0.70 & 0.82 & 0.58 & 0.74 & 0.78 & 0.66 \\
\hline $\mathrm{Fe}^{2+}$ & 1.28 & 1.25 & 1.20 & 1.13 & 1.31 & 1.19 & 1.18 & 1.30 \\
\hline $\mathrm{Cr}$ & 0.00 & 0.00 & 0.00 & 0.00 & 0.00 & 0.00 & 0.01 & 0.01 \\
\hline $\mathrm{Mn}$ & 0.02 & 0.03 & 0.02 & 0.03 & 0.03 & 0.02 & 0.02 & 0.03 \\
\hline $\mathrm{Mg}$ & 3.41 & 3.41 & 3.40 & 3.46 & 3.41 & 3.46 & 3.50 & 3.50 \\
\hline $\mathrm{Ba}$ & 0.01 & 0.01 & 0.01 & 0.01 & 0.01 & 0.01 & 0.01 & 0.01 \\
\hline $\mathrm{Ca}$ & 0.00 & 0.00 & 0.01 & 0.01 & 0.04 & 0.00 & 0.01 & 0.03 \\
\hline $\mathrm{Na}$ & 0.02 & 0.01 & 0.02 & 0.03 & 0.02 & 0.03 & 0.02 & 0.01 \\
\hline $\mathrm{K}$ & 1.94 & 1.91 & 1.90 & 1.87 & 1.88 & 1.86 & 1.89 & 1.91 \\
\hline $\mathrm{F}$ & 0.24 & 0.29 & 0.23 & 0.14 & 0.29 & 0.19 & 0.49 & 0.24 \\
\hline $\mathrm{Cl}$ & 0.17 & 0.16 & 0.15 & 0.16 & 0.15 & 0.15 & 0.15 & 0.17 \\
\hline
\end{tabular}

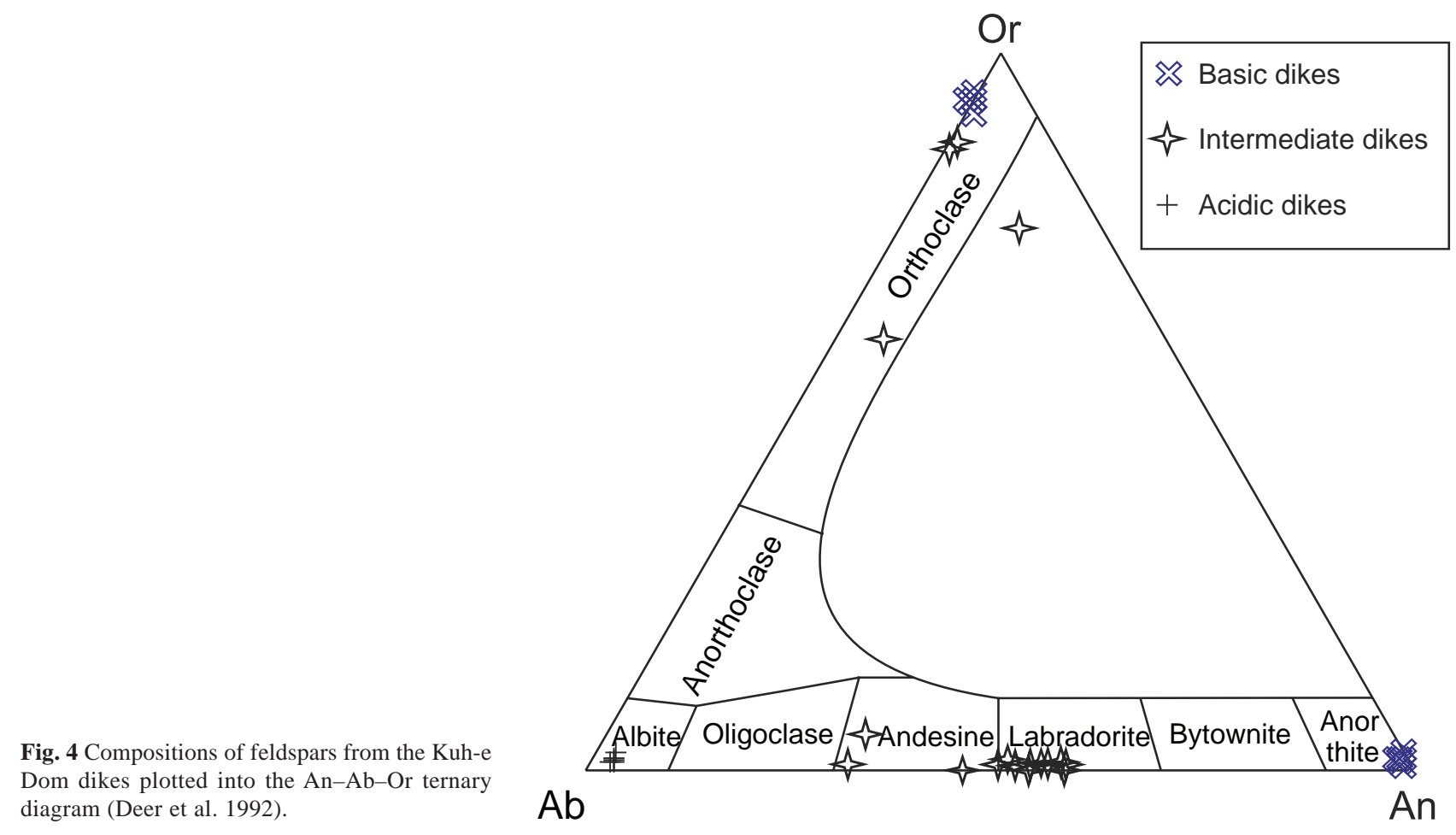




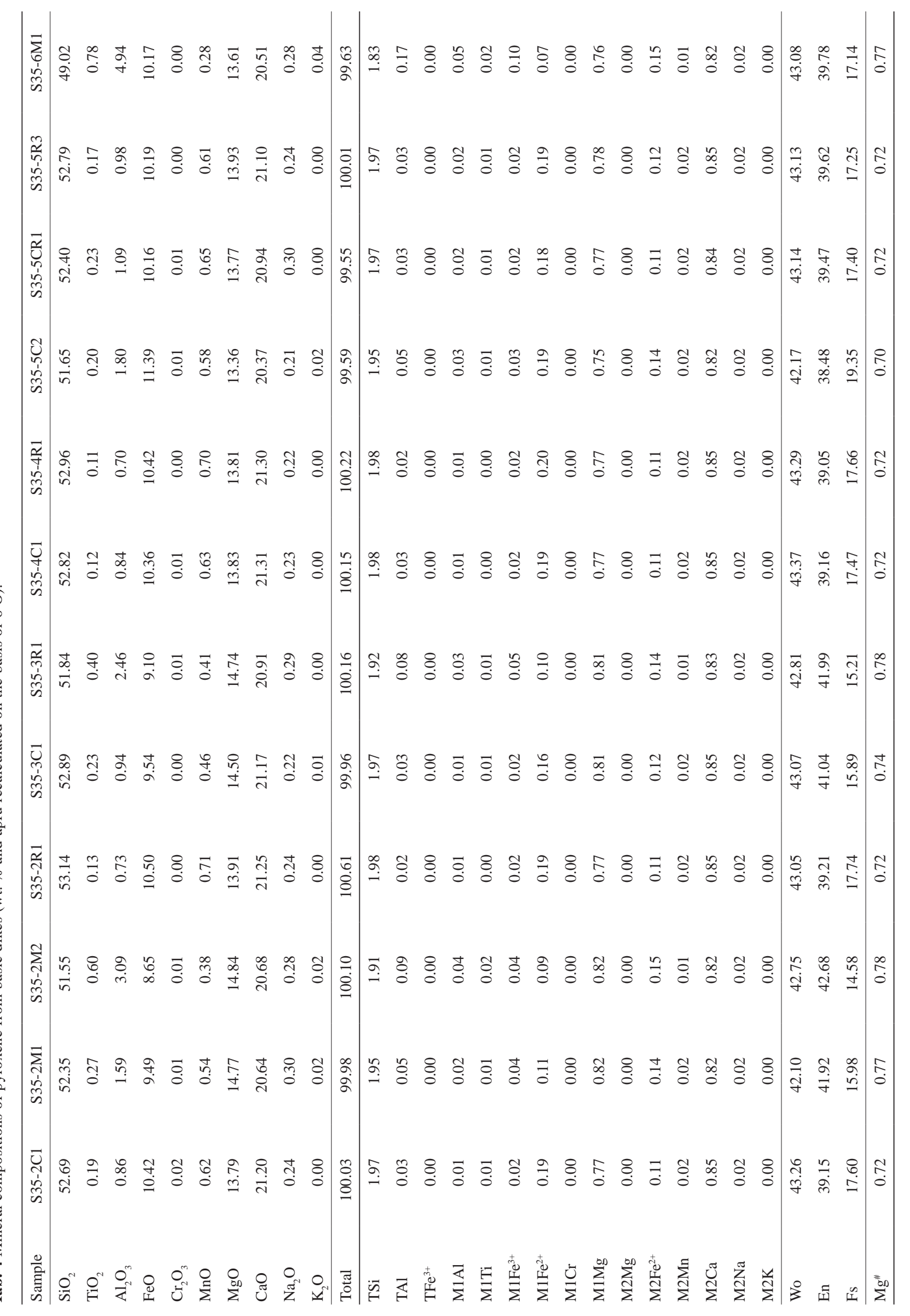




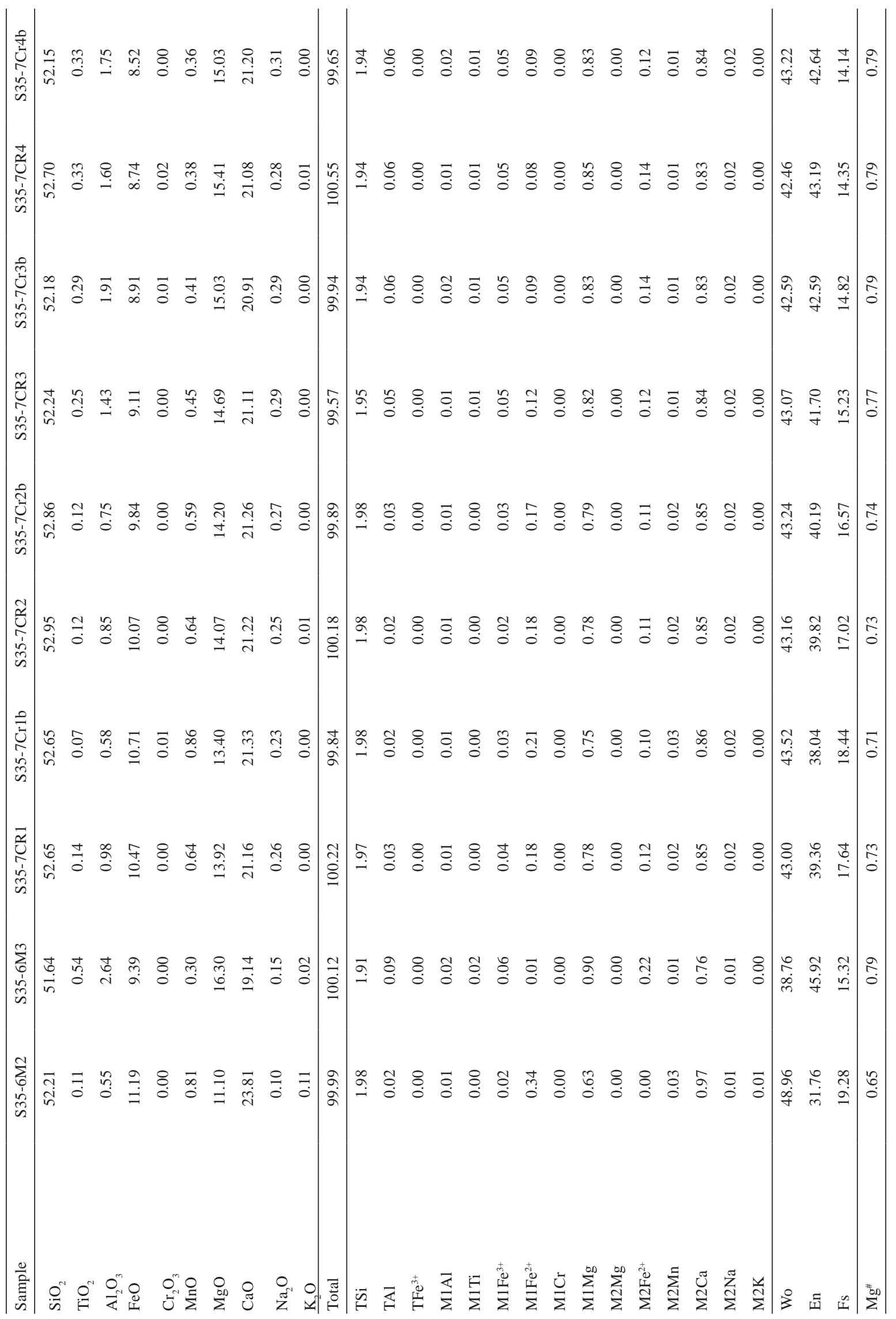




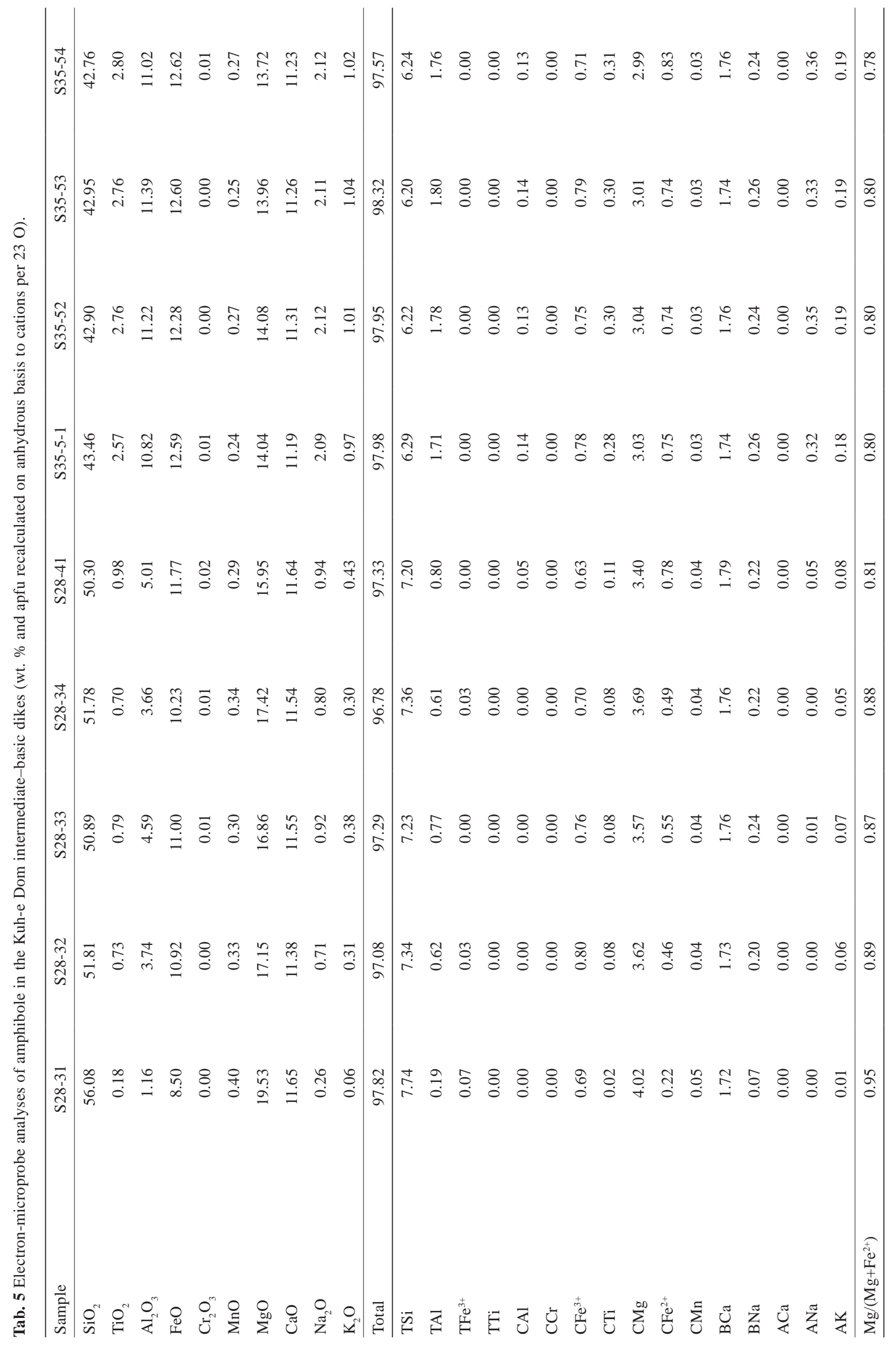


Tab. 6 The whole-rock geochemical data from acidic and intermediate-basic dikes (major elements in wt. \%, trace elements in ppm)

\begin{tabular}{|c|c|c|c|c|c|c|c|c|c|c|c|c|c|}
\hline Sample & $\begin{array}{r}\text { H23 } \\
\operatorname{dik} \text { A }\end{array}$ & $\begin{array}{c}\text { F12 } \\
\operatorname{dik} \text { A }\end{array}$ & $\begin{array}{c}\mathrm{F} 2 \\
\operatorname{dik} \mathrm{A}\end{array}$ & $\begin{array}{c}\text { H3 } \\
\operatorname{dik} \text { A }\end{array}$ & $\begin{array}{c}\text { F42 } \\
\operatorname{dik} \text { A }\end{array}$ & $\begin{array}{c}\text { H7 } \\
\operatorname{dik} \text { A }\end{array}$ & $\begin{array}{c}\text { S65 } \\
\operatorname{dik} \text { A }\end{array}$ & $\begin{array}{c}\text { S15 } \\
\operatorname{dik} B\end{array}$ & $\begin{array}{c}\text { F54 } \\
\text { dik B }\end{array}$ & $\begin{array}{c}\text { S10 } \\
\text { dik B }\end{array}$ & $\begin{array}{c}\text { S26 } \\
\text { dik B }\end{array}$ & $\begin{array}{c}\text { S28 } \\
\text { dik B }\end{array}$ & $\begin{array}{c}\text { S35 } \\
\operatorname{dik} \text { B }\end{array}$ \\
\hline $\mathrm{SiO}_{2}$ & 75.50 & 70.45 & 76.90 & 75.20 & 72.69 & 68.30 & 65.30 & 59.60 & 52.71 & 51.10 & 46.50 & 49.30 & 54.10 \\
\hline $\mathrm{TiO}_{2}$ & 0.13 & 0.29 & 0.08 & 0.06 & 0.24 & 0.32 & 0.39 & 0.51 & 1.05 & 0.66 & 0.91 & 0.96 & 0.72 \\
\hline $\mathrm{Al}_{2} \mathrm{O}_{3}$ & 12.45 & 14.1 & 12.32 & 11.9 & 13.33 & 14.85 & 15.15 & 15.2 & 16.58 & 16.85 & 16.5 & 15.95 & 17.60 \\
\hline $\mathrm{Fe}_{2} \mathrm{O}_{3}$ & 0.84 & 1.31 & 0.57 & 0.32 & 1.63 & 1.16 & 1.43 & 1.94 & 3.107 & 3.43 & 2.21 & 2.97 & 2.36 \\
\hline $\mathrm{FeO}$ & 0.93 & 1.59 & 0.57 & 0.34 & 1.69 & 1.29 & 1.54 & 2.82 & 4.56 & 4.18 & 3.22 & 4.64 & 2.27 \\
\hline $\mathrm{MnO}$ & 0.02 & 0.09 & 0.03 & 0.03 & 0.03 & 0.04 & 0.09 & 0.14 & 0.17 & 0.17 & 0.13 & 0.17 & 0.21 \\
\hline MgO & 0.33 & 0.66 & 0.16 & 0.12 & 0.96 & 1.12 & 1.75 & 2.03 & 3.22 & 3.47 & 4.27 & 3.01 & 2.77 \\
\hline $\mathrm{CaO}$ & 1.02 & 3.96 & 0.63 & 1.82 & 0.56 & 1.24 & 2.05 & 3.90 & 8.05 & 4.48 & 8.43 & 9.09 & 6.15 \\
\hline $\mathrm{Na}_{2} \mathrm{O}$ & 3.06 & 1.38 & 3.53 & 0.17 & 0.66 & 3.98 & 4.68 & 2.37 & 2.77 & 4.76 & 6.13 & 2.82 & 1.00 \\
\hline $\mathrm{K}_{2} \mathrm{O}$ & 4.78 & 5.87 & 5.14 & 8.25 & 7.93 & 4.22 & 3.96 & 3.93 & 3.82 & 3.61 & 0.89 & 3.45 & 9.35 \\
\hline $\mathrm{P}_{2} \mathrm{O}_{5}$ & 0.02 & 0.10 & 0.01 & 0.01 & 0.08 & 0.11 & 0.13 & 0.17 & 0.40 & 0.22 & 0.30 & 0.38 & 0.25 \\
\hline LOI & 0.80 & 0.08 & 0.12 & 1.73 & 0.15 & 2.40 & 3.20 & 6.04 & 3.40 & 6.67 & 9.78 & 5.58 & 2.98 \\
\hline Total & 99.88 & 99.89 & 100.1 & 99.95 & 99.95 & 99.03 & 99.67 & 98.65 & 99.84 & 99.6 & 99.26 & 98.33 & 99.76 \\
\hline $\mathrm{Rb}$ & 179 & 140 & 165 & 209 & 173 & 127 & 98 & 112 & 124 & 83 & 22 & 116 & 252 \\
\hline $\mathrm{Sr}$ & 103.50 & 54.10 & 41.40 & 29.60 & 34.60 & 77.80 & 83.90 & 97.60 & 498.00 & 125.00 & 169.50 & 527.00 & 462.00 \\
\hline $\mathrm{Ga}$ & 13.10 & * & * & 10.50 & 13.40 & 15.10 & 14.80 & 15.20 & 17.70 & 18.00 & 16.80 & 18.90 & 16.00 \\
\hline $\mathrm{Nb}$ & 14.80 & 10.83 & 17.63 & 12.10 & 14.50 & 15.60 & 12.60 & 13.80 & 23.60 & 10.40 & 20.3 & 24.50 & 13.00 \\
\hline $\mathrm{Hf}$ & 3.60 & * & * & 2.30 & 3.50 & 4.10 & 4.40 & 4.30 & 4.90 & 3.30 & 3.50 & 5.60 & 3.40 \\
\hline $\mathrm{Zr}$ & 107 & 129 & 101 & 62 & 129 & 148 & 163 & 155 & 191 & 124 & 149 & 223 & 123 \\
\hline $\mathrm{Y}$ & 13.70 & 20.50 & 22.60 & 13.90 & 13.70 & 15.50 & 16.10 & 18.10 & 22.90 & 22.40 & 29.10 & 23.50 & 20.70 \\
\hline Th & 26.9 & 14.41 & 47.60 & 14.55 & 15.45 & 14.40 & 14.40 & 13.30 & 14.90 & 9.36 & 5.65 & 16.00 & 10.80 \\
\hline U & 5.36 & $*$ & $*$ & 3.09 & 2.95 & 4.32 & 3.59 & 3.37 & 3.94 & 2.65 & 2.22 & 4.55 & 3.35 \\
\hline $\mathrm{Cr}$ & 10 & 0 & 0 & 10 & 10 & 10 & 10 & 10 & 230 & 20 & 370 & 160 & 10 \\
\hline $\mathrm{Ni}$ & 7 & 2 & 2 & 6 & 5 & 5 & 7 & 6 & 63 & 10 & 109 & 50 & 11 \\
\hline Co & 4.60 & $*$ & $*$ & 0.50 & 3.90 & 4.80 & 4.30 & 9.60 & 23.80 & 16.20 & 11.10 & 22.10 & 9.40 \\
\hline V & 19 & $*$ & $*$ & 5 & 22 & 43 & 62 & 96 & 196 & 167 & 168 & 226 & 206 \\
\hline $\mathrm{Zn}$ & 23 & $*$ & $*$ & 7 & 20 & 21 & 30 & 27 & 91 & 375 & 46 & 44 & 121 \\
\hline Тa & 1.80 & $*$ & $*$ & 1.50 & 1.40 & 1.30 & 1.10 & 1.20 & 1.30 & 0.70 & 1.50 & 1.60 & 0.90 \\
\hline Se & $*$ & $*$ & $*$ & $*$ & $*$ & $*$ & 1.10 & 1.20 & $*$ & 0.70 & 1.50 & 1.60 & 0.90 \\
\hline Cs & 3.81 & $*$ & $*$ & 2.40 & 1.19 & 3.01 & 1.19 & 5.78 & 5.76 & 0.50 & 0.23 & 5.12 & 1.34 \\
\hline $\mathrm{La}$ & 33.60 & $*$ & $*$ & 30.20 & 28.80 & 34.10 & 35.10 & 32.70 & 35.50 & 23.40 & 20.30 & 35.70 & 27.00 \\
\hline $\mathrm{Ce}$ & 58.20 & 40.50 & 70.40 & 52.70 & 53.00 & 60.30 & 63.20 & 58.70 & 66.90 & 46.10 & 39.50 & 67.70 & 52.40 \\
\hline $\operatorname{Pr}$ & 5.66 & * & * & 5.58 & 5.67 & 6.18 & 6.75 & 6.43 & 7.73 & 5.44 & 4.81 & 7.80 & 6.25 \\
\hline $\mathrm{Nd}$ & 17.60 & $*$ & * & 18.40 & 18.20 & 20.30 & 23.70 & 22.20 & 28.10 & 21.20 & 19.10 & 29.70 & 23.80 \\
\hline $\mathrm{Sm}$ & 2.92 & $*$ & $*$ & 3.48 & 3.13 & 3.51 & 4.03 & 4.21 & 5.77 & 4.31 & 4.16 & 5.86 & 4.92 \\
\hline Eu & 0.50 & $*$ & $*$ & 0.74 & 0.51 & 0.85 & 0.79 & 1.04 & 1.47 & 1.13 & 1.19 & 1.43 & 1.28 \\
\hline $\mathrm{Gd}$ & 2.67 & $*$ & $*$ & 2.90 & 2.82 & 3.34 & 3.8 & 4.13 & 5.43 & 4.54 & 4.69 & 5.84 & 4.85 \\
\hline $\mathrm{Tb}$ & 0.37 & $*$ & $*$ & 0.41 & 0.38 & 0.45 & 0.47 & 0.59 & 0.79 & 0.70 & 0.78 & 0.81 & 0.68 \\
\hline Dy & 2.26 & $*$ & $*$ & 2.34 & 2.24 & 2.51 & 2.62 & 3.29 & 4.38 & 4.00 & 5.02 & 4.39 & 3.96 \\
\hline Ho & 0.46 & $*$ & $*$ & 0.50 & 0.43 & 0.53 & 0.58 & 0.65 & 0.82 & 0.80 & 1.08 & 0.89 & 0.78 \\
\hline $\mathrm{Er}$ & 1.47 & $*$ & $*$ & 1.66 & 1.56 & 1.71 & 2.00 & 2.02 & 2.44 & 2.45 & 3.40 & 2.50 & 2.31 \\
\hline $\mathrm{Tm}$ & 0.25 & $*$ & $*$ & 0.26 & 0.23 & 0.26 & 0.33 & 0.29 & 0.34 & 0.37 & 0.49 & 0.35 & 0.34 \\
\hline $\mathrm{Yb}$ & 1.73 & $*$ & $*$ & 1.85 & 1.55 & 1.87 & 2.20 & 2.01 & 2.13 & 2.40 & 3.02 & 2.24 & 2.14 \\
\hline $\mathrm{Lu}$ & 0.28 & $*$ & $*$ & 0.30 & 0.25 & 0.32 & 0.38 & 0.33 & 0.35 & 0.36 & 0.45 & 0.36 & 0.33 \\
\hline $\mathrm{A} / \mathrm{CNK}$ & 1.03 & 0.89 & 0.98 & 0.95 & 1.25 & 1.11 & 0.96 & 1.00 & 0.58 & 0.85 & 0.63 & 0.64 & 0.77 \\
\hline (La/Yb)n & 13.19 & * & $*$ & 11.09 & 12.62 & 12.39 & 10.84 & 11.05 & 11.32 & 6.62 & 4.57 & 10.83 & 8.57 \\
\hline$\left(\mathrm{Eu} / \mathrm{Eu}^{*}\right)$ & 0.54 & $*$ & $*$ & 0.69 & 0.51 & 0.75 & 0.61 & 0.75 & 0.79 & 0.77 & 0.82 & 0.74 & 0.79 \\
\hline $\mathrm{Ce} / \mathrm{Yb}$ & 33.64 & $*$ & $*$ & 28.49 & 34.19 & 32.25 & 28.73 & 29.20 & 31.41 & 19.21 & 13.08 & 30.22 & 24.49 \\
\hline $\mathrm{Ta} / \mathrm{Yb}$ & 1.04 & $*$ & $*$ & 0.81 & 0.90 & 0.70 & 0.50 & 0.60 & 0.61 & 0.29 & 0.50 & 0.71 & 0.42 \\
\hline $\mathrm{Zr} / \mathrm{Sm}$ & 36.64 & $*$ & $*$ & 17.82 & 41.21 & 42.17 & 40.45 & 36.82 & $*$ & $*$ & $*$ & $*$ & * \\
\hline $\mathrm{Nb} / \mathrm{Ta}$ & 8.22 & $*$ & $*$ & 8.07 & 10.36 & 12.00 & 11.45 & 11.50 & $*$ & $*$ & * & $*$ & * \\
\hline $\mathrm{Ba} / \mathrm{Rb}$ & $*$ & $*$ & $*$ & $*$ & $*$ & * & $*$ & * & 4.78 & 6.37 & 7.28 & 4.81 & 10.63 \\
\hline $\mathrm{Rb} / \mathrm{Sr}$ & $*$ & $*$ & $*$ & $*$ & $*$ & $*$ & $*$ & $*$ & 0.25 & 0.66 & 0.13 & 0.22 & 0.55 \\
\hline
\end{tabular}

dik A: acidic dikes, dik B: basic-intermediate dikes

* - not determined 

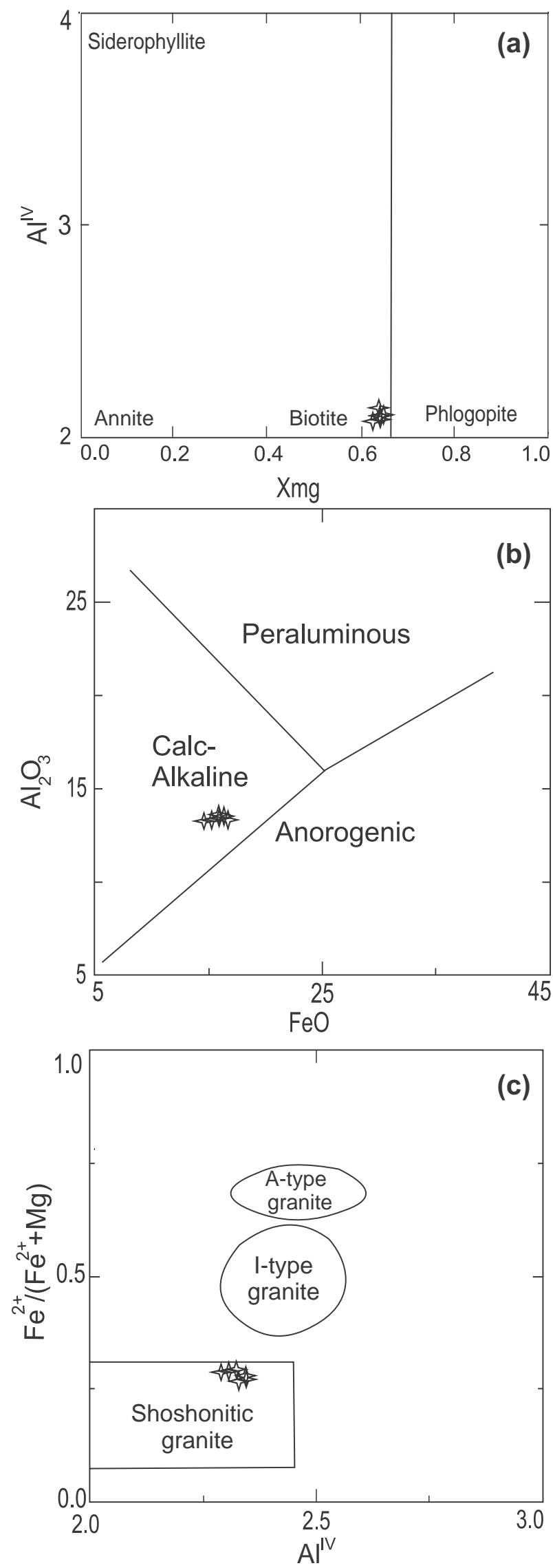

The clinopyroxene of the basic dikes corresponds to diopside-augite (Fig. 6a). The $\mathrm{Mg}^{\#}$ of the clinopyroxene phenocrysts vary a little around 0.7 , and the average value correlates well with the whole-rock compositions. Some phenocrysts show oscillatory zoning in $\mathrm{Al}, \mathrm{Ti}$ and $\mathrm{Mg}$ contents, consistent with the crystal growth and/or the element diffusion in liquids with different compositions and temperatures (Ridolfi et al. 2008). According to the classification of Le Bas (1962), the pyroxenes show subalkaline affinity (Fig. 6b). The association of diopside-augite clinopyroxene is characteristic of shoshonitic rocks as well (Morrison 1980).

According to classification scheme of Leake et al. (1997) and Hawthorne and Oberti (2007), amphiboles of intermediate dikes are mainly magnesiohornblendes, whereby those from basic dikes correspond to magnesiohastingsite $\left({ }^{\mathrm{VI}} \mathrm{Al}<\mathrm{Fe}^{3+}\right.$; Fig. 7a). Tremolite probably crystallized as a subsolidus phase since it falls outside the limit of igneous amphibole.

Based on earlier studies (Chappell and White 1974; White and Chappell 1983; Wyborn et al. 1981; Clemens and Wall 1984), the presence of calcic amphiboles in granitoid rocks indicates I-type affinity. Fig. 7b shows that the compositions of amphibole from intermediate dikes are consistent with crystallization from subalkaline magmas, while those from the basic dikes have higher $\mathrm{TiO}_{2}$ and $\mathrm{Al}_{2} \mathrm{O}_{3}$ contents and thus fall into the transitional subalkaline-alkaline domain (Molina et al. 2009). The compositional differences between amphiboles from both rock types may imply a difference in the physicochemical conditions of magmas from which they crystallized.

\section{Geochemistry}

Geochemical compositions of the studied dikes are listed in Tab. 6. Using the $\mathrm{Zr} / \mathrm{TiO}_{2}$ vs. silica diagram (Winchester and Floyd 1977), the silicic dikes can be classified as rhyolite, rhyodacite and dacite, while the intermediate-basic rocks show phonolite, basanite and trachyandesite composition (Fig. 8).

In the $\mathrm{A} / \mathrm{CNK}\left[\right.$ molar $\left.\mathrm{Al}_{2} \mathrm{O}_{3} /\left(\mathrm{CaO}+\mathrm{Na}_{2} \mathrm{O}+\mathrm{K}_{2} \mathrm{O}\right)\right]$ versus $\mathrm{Zr}+\mathrm{Nb}+\mathrm{Ce}+\mathrm{Y}$ diagram (Condie et al.1999; Fig. 9), the acidic dikes plot as metaluminous to slightly peraluminous. The intermediate-basic dikes fall in the metaluminous field, some far from the discrimination line of the metaluminous and peraluminous domains. This diagram shows that the studied dikes are of I- rather than A-type nature.

Fig. 5 Biotite compositions from intermediate dikes. $\mathbf{a}$ - classification diagram of Rieder et al. (1998). b - $\mathrm{Al}_{2} \mathrm{O}_{3}-\mathrm{FeO}_{\mathrm{T}}$ diagram (AbdelRahman 1994) discriminating the likely geotectonic setting of biotites. c $-\mathrm{Fe}^{2+} /\left(\mathrm{Fe}^{2+}+\mathrm{Mg}\right)$ vs. $\mathrm{Al}^{\mathrm{IV}}$ diagram (Jiang et al. 2002). 


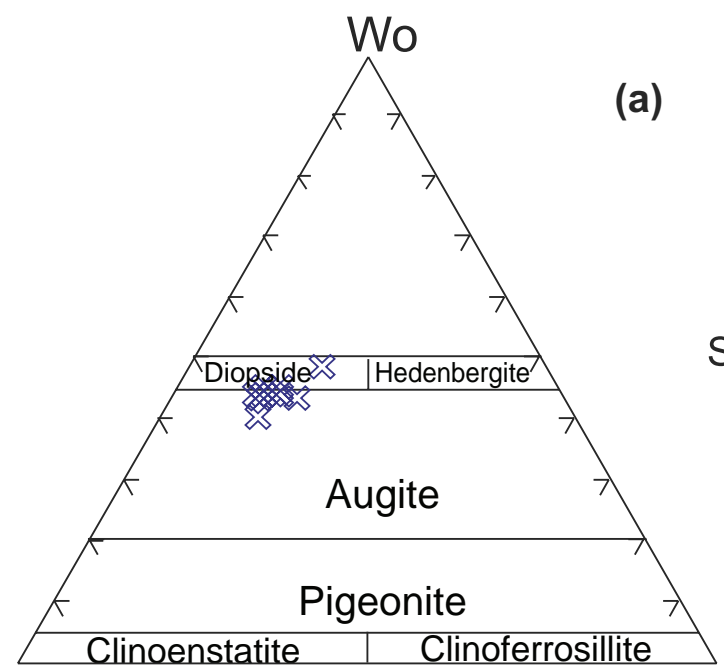

En
Fs

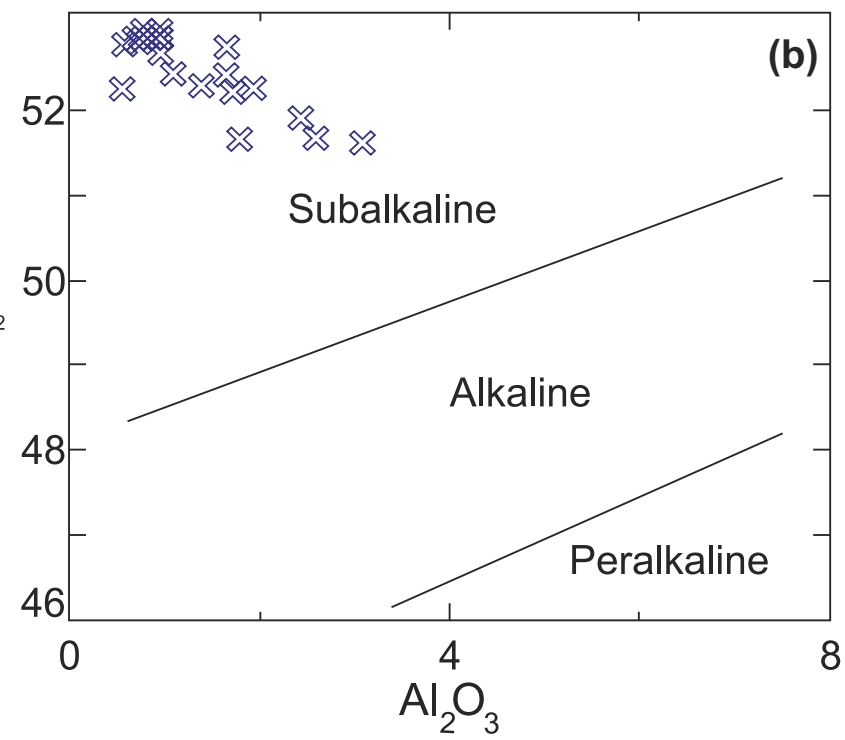

(b)

Fig. 6 Composition of clinopyroxenes from the basic dikes. a - Wo-En-Fs classification diagram (Morimoto 1988). $\mathbf{b}-\mathrm{Al}_{2} \mathrm{O}_{3}-\mathrm{SiO}_{2}$ diagram (Le Bas 1962) discriminating the nature of the pyroxene host rocks.

(a)

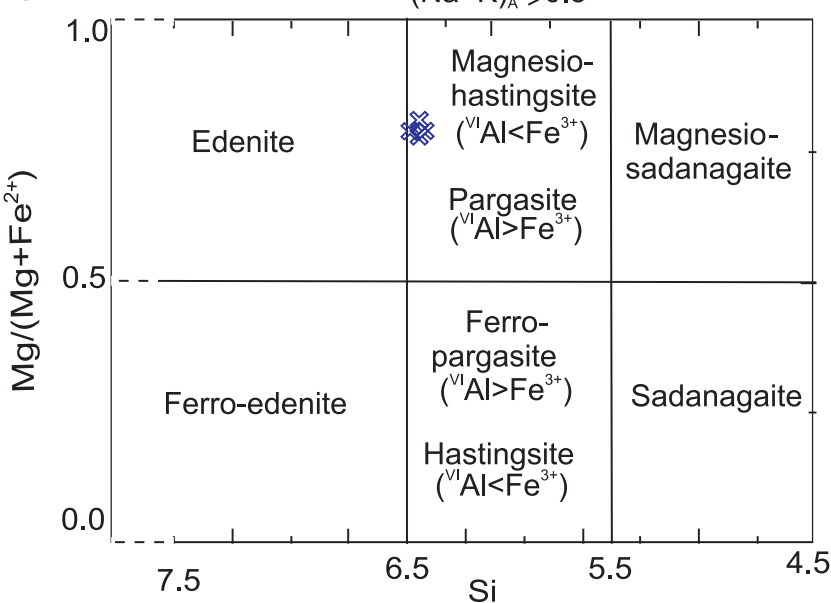

(c)

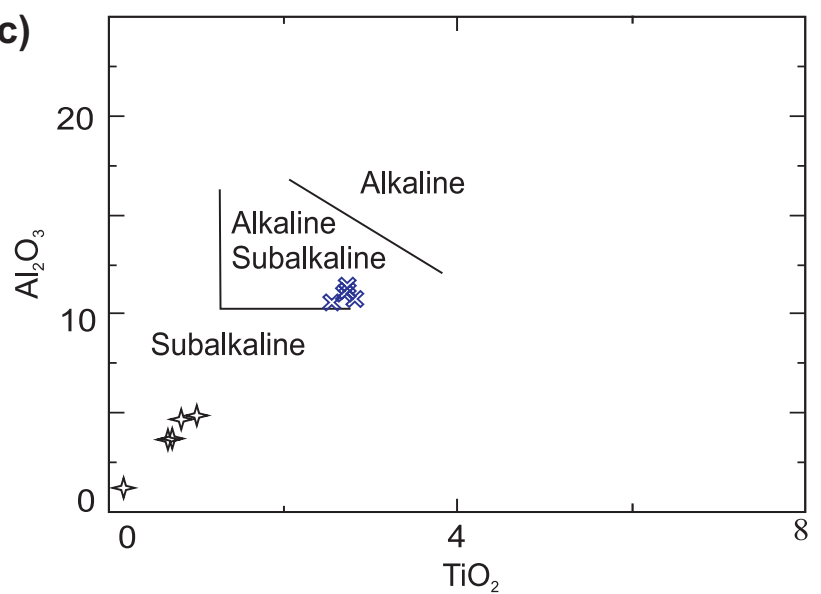

(b)

$(\mathrm{Na}+\mathrm{K})_{\mathrm{A}}<0.5$

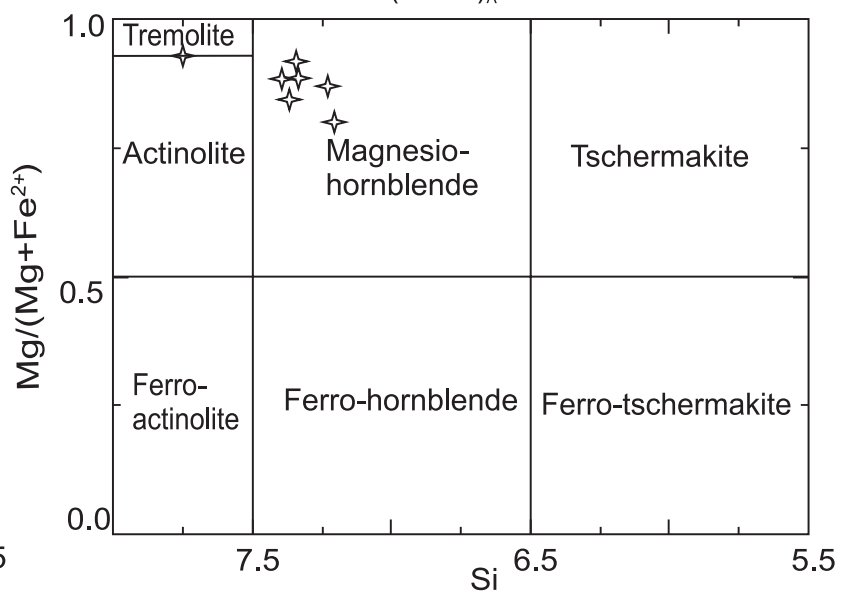

Fig. 7a-b Classification diagram for amphiboles (Leake et al. 1997; Hawthorne and Oberti 2007). c - Composition of studied amphiboles in the $\mathrm{Al}_{2} \mathrm{O}_{3}$ vs. $\mathrm{TiO}_{2}$ binary diagram (Molina et al. 2009). 


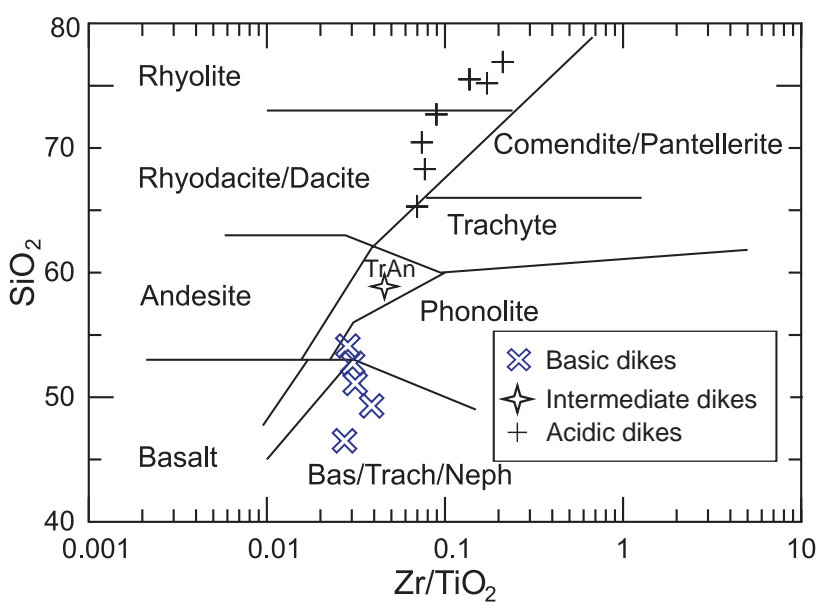

Fig. $8 \mathrm{Zr} / \mathrm{TiO}_{2}$ vs. $\mathrm{SiO}_{2}$ diagram for nomenclature of the studied dikes (Winchester and Floyd 1977).

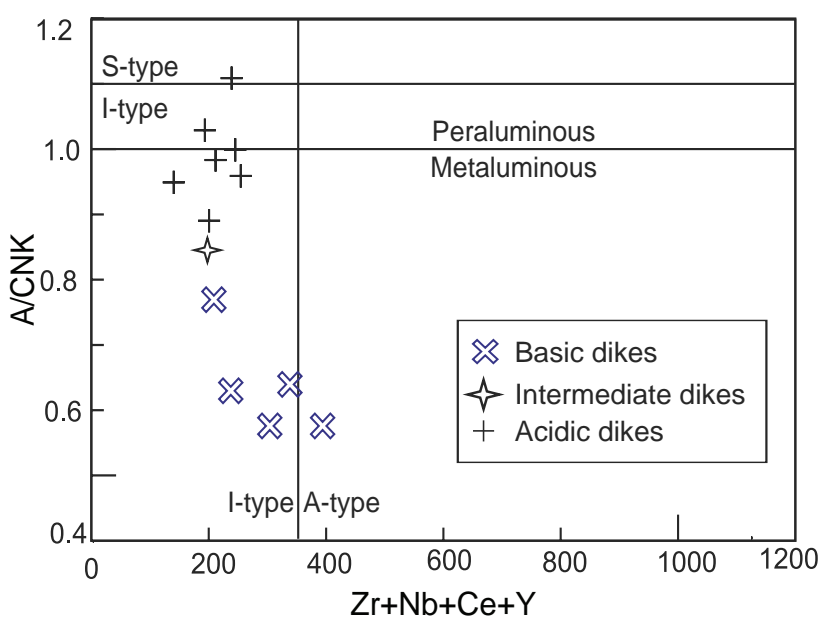

Fig. $9 \mathrm{~A} / \mathrm{CNK}\left[\mathrm{Al}_{2} \mathrm{O}_{3} /\left(\mathrm{CaO}+\mathrm{Na}_{2} \mathrm{O}+\mathrm{K}_{2} \mathrm{O}\right)\right.$ molecular ratio] vs. $\mathrm{Zr}+\mathrm{Nb}$ $+\mathrm{Ce}+\mathrm{Y}$ (Condie et al. 1999), showing the nature of the studied dikes.
The Th-Co diagram (Hastie et al. 2007; Fig. 10a) has been designed to replace a classic $\mathrm{SiO}_{2}-\mathrm{K}_{2} \mathrm{O}$ plot of Peccerillo and Taylor (1976) for altered igneous rocks. The studied dikes plot in the high-K calc-alkaline and shoshonitic field. Likewise, in $\mathrm{Ce} / \mathrm{Yb}$ versus $\mathrm{Ta} / \mathrm{Yb}$ diagram (Pearce 1982; Fig. 10b), most of the samples fall in the shoshonitic domain.

All indicators such as (1) high alkali content $\left(\mathrm{K}_{2} \mathrm{O}+\right.$ $\mathrm{Na}_{2} \mathrm{O}>5$ wt. \%: Turner et al. 1996), (2) LILE enrichment relative to HFSE, and (3) low $\mathrm{TiO}_{2}$ content $(<1.2$ wt. \%: Morrison 1980) document that the Kuh-e Dom dikes have shoshonitic affinity. Furthermore, $\mathrm{Zr} / \mathrm{Hf}$ ratios range between 27 and 42, consistent with similar ratios in shoshonitic igneous rocks (Carmichael et al. 1996; Müller and Forrestal 1998).

In chondrite-normalized REE plots (Sun and McDonough 1989; Fig. 11a), the acidic dikes display lower HREE contents and higher degree of LREE/HREE enrichment than the intermediate-basic dikes do. The silicic dikes (Fig. 11b) show nearly parallel trends, with moderate LREE enrichment $\left((\mathrm{La} / \mathrm{Yb})_{\mathrm{n}}=11-13\right)$ and sizeable negative $\mathrm{Eu}$ anomalies $\left(\mathrm{Eu} / \mathrm{Eu}^{*}=0.51-0.75\right)$. Also the REE patterns for intermediate-basic dikes are subparallel, but the negative Eu anomalies are shallower $\left(\mathrm{Eu} / \mathrm{Eu}^{*}=\right.$ $0.79-0.90)$ and the patterns less steep $\left((\mathrm{La} / \mathrm{Yb})_{\mathrm{n}}=5-11\right)$.

Primitive mantle-normalized (Sun and McDonough 1989) patterns for silicic dikes (Fig. 12a) are largely parallel and show LILE enrichment relative to HFSE, as well as negative anomalies of $\mathrm{Nb}\left(\mathrm{Nb} / \mathrm{Nb}^{*} \sim 0.12\right)$, $\mathrm{Ta}\left(\mathrm{Ta} / \mathrm{Ta}^{*} \sim 0.3\right)$, $\mathrm{Sr}(\mathrm{Sr} / \mathrm{Sr} * \sim 0.13), \mathrm{P}\left(\mathrm{P} / \mathrm{P}^{*} \sim 0.19\right)$ and $\mathrm{Ti}\left(\mathrm{Ti} / \mathrm{Ti}^{*} \sim 0.14\right)$. Similar features show the intermediate-basic dikes (Fig. 12b) even though the negative anomalies are less conspicuous $\left(\mathrm{Nb} / \mathrm{Nb}^{*} \sim 0.25, \mathrm{Ta} / \mathrm{Ta} *\right.$ $\sim 0.3$ and $\mathrm{Ti} / \mathrm{Ti}^{*} \sim 0.48$ ). The Sr contents of these dikes (except two samples which are altered to spilite) do not show any depletion.
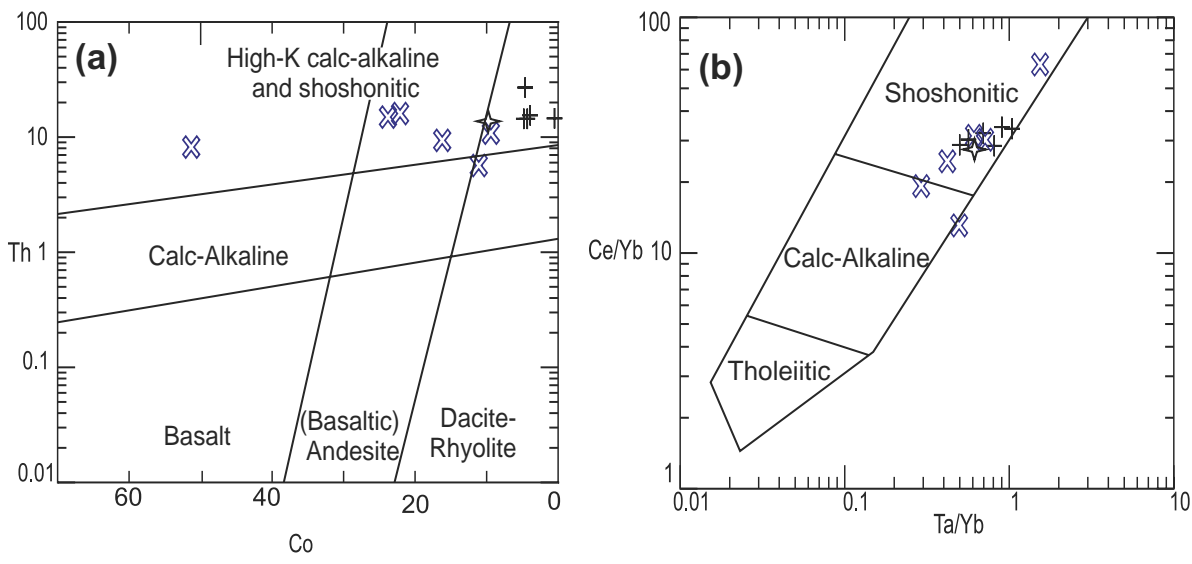

Fig. 10 Co vs. Th (Hastie et al. 2007) (a) and Ce/Yb vs. Ta/Yb diagram (Pearce 1982) (b) which document the shoshonitic affinity of the studied dikes.

\section{Discussion}

\subsection{Dikes origin}

\subsubsection{Silicic dikes}

We discuss three models for the generation of silicic dikes: (1) fractional crystallization of basic magma parental to the intermediate-basic dikes with, or without, assimilation, (2) origin from residual melt during the final stages of crystallization of the granite host and (3) partial melting of lower crust. 
Were the silicic dikes generated by fractionation of more basic melts, the REE patterns of all rock types should be parallel but they are not (Fig. 11a). Moreover, the relatively large volume of silicic dikes compared to intermediate-basic dikes argues against such a model.

Silicic dikes have HREE contents significantly lower, but their LREE contents fall within, the span observed in their igneous host rocks (shaded area in Fig. 11b). Assuming that the silicic dikes are products of evolved residual granitic melt, they should show higher LREE content relative to the parental magma (Rollinson 1993) due to the remarkably greater abundance of amphibole and biotite in the host granite, but this is not the case (Fig. 11b).

In primitive mantle-normalized spider plots, the silicic dikes show negative anomalies in $\mathrm{Ti}, \mathrm{P}, \mathrm{Sr}, \mathrm{Nb}$ and $\mathrm{Ta}$. These are important features of fractionated granitic magmas, e.g. in the Lachlan Orogenic Belt (White and Chappell 1983; Chappell and White 1992). Experimental studies show that silica-rich ( $>56$ wt. $\% \mathrm{SiO}_{2}$ ) and high$\mathrm{K}$, I type granitoid magmas can be derived from partial melting of older metaigneous rocks in the continental crust (Roberts and Clemens, 1993; Patiño Douce and McCarthy 1998; Yardley and Valley 1997). Because of their low $\mathrm{K}_{2} \mathrm{O}$ contents, metabasaltic rocks of all kinds are unsuitable as sources unlike the hydrous, calc-alkaline to high-K calc-alkaline, mafic to intermediate metamorphic rocks (Roberts and Clemens 1993).

Melting of pelitic crust alone could not produce metaluminous magma. There is no evidence on the extensive magmatic fractionation among the samples. In the $\mathrm{Al}_{2} \mathrm{O}_{3}$ $+\mathrm{FeOt}+\mathrm{MgO}+\mathrm{TiO}_{2}$ vs. $\mathrm{Al}_{2} \mathrm{O}_{3} /\left(\mathrm{FeOt}+\mathrm{MgO}+\mathrm{TiO}_{2}\right) \mathrm{di}-$ agram (Fig. 13; Patiño Douce 1999), most of the samples plot in the field of greywacke-derived melts. Therefore it is seen that partial melting of a quartzo-feldspathic crust was probably the main mechanism for formation of silicic dikes.

\subsubsection{Intermediate-basic dikes}

The more basic dikes show shoshonitic affinity with LILE and LREE enrichment. Some authors interpret subcontinental lithospheric mantle, or asthenospheric mantle enriched in incompatible elements by earlier subduction, as the principal sources of shoshonitic and associated high-K calc-alkaline magmas (Aldanmaz et al. 2000; Seghedi et al. 2004). Others have emphasized magma fractionation and crustal assimilation as the dominant processes (Meen 1987; Feeley and Cosca 2003).

Experimental data indicate that the K-rich magma could not form by low-degree (2-2.5\%) partial melting of lherzolite at less than $2-3 \mathrm{GPa}(70-100 \mathrm{~km})$ as it can
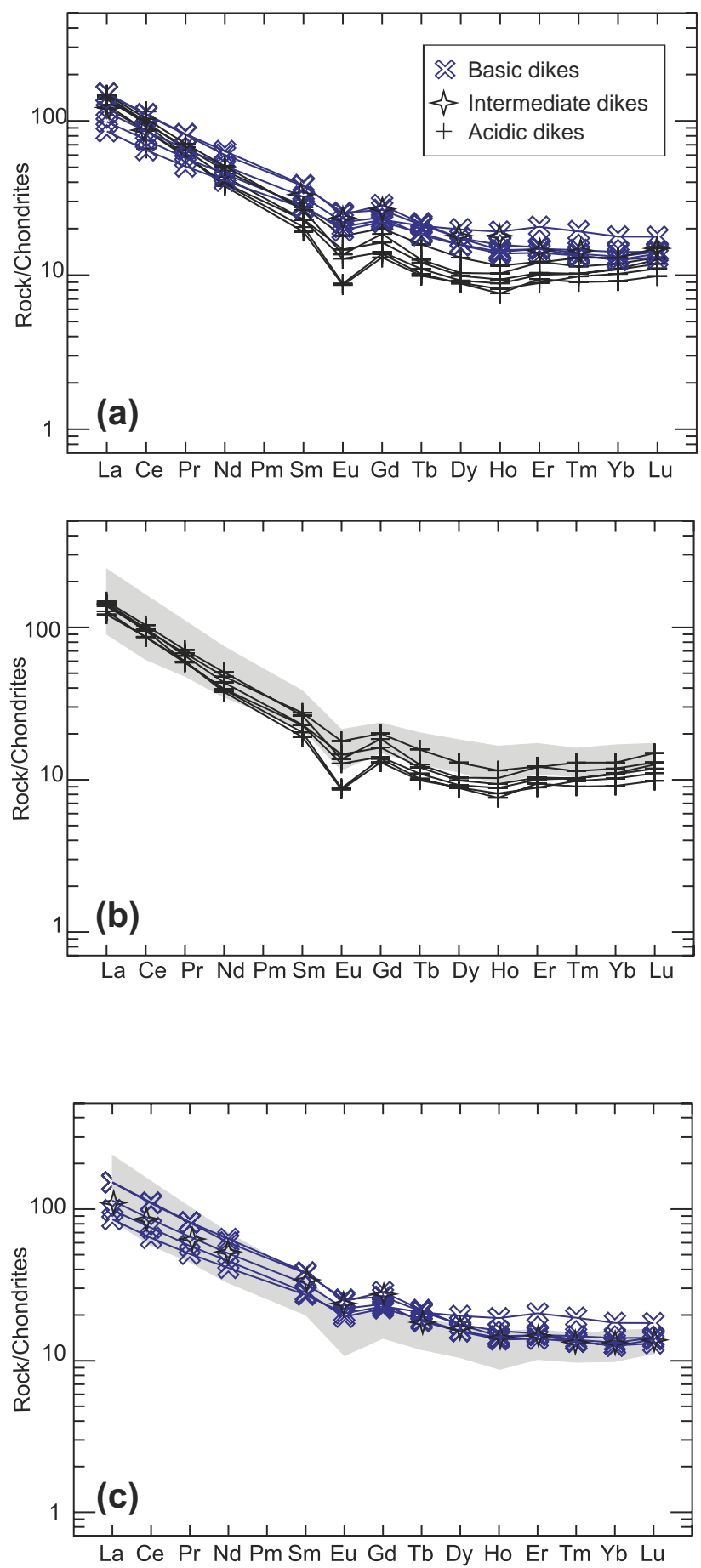

Fig. 11 Chondrite-normalized rare earth elements patterns of Kuh-e Dom dikes (Sun and McDonough 1989) (a) and the comparison of acidic (b) and intermediate-basic dikes (c) with the host-rock patterns (gray field).

only yield basanitic magma with $\mathrm{SiO}_{2}$ contents inconsistent with the criteria for shoshonites (Wang et al. 1991). Crustal contamination alone also cannot generate K-rich magma from partial melt originally derived from pyrolite (Wang et al. 1991). 

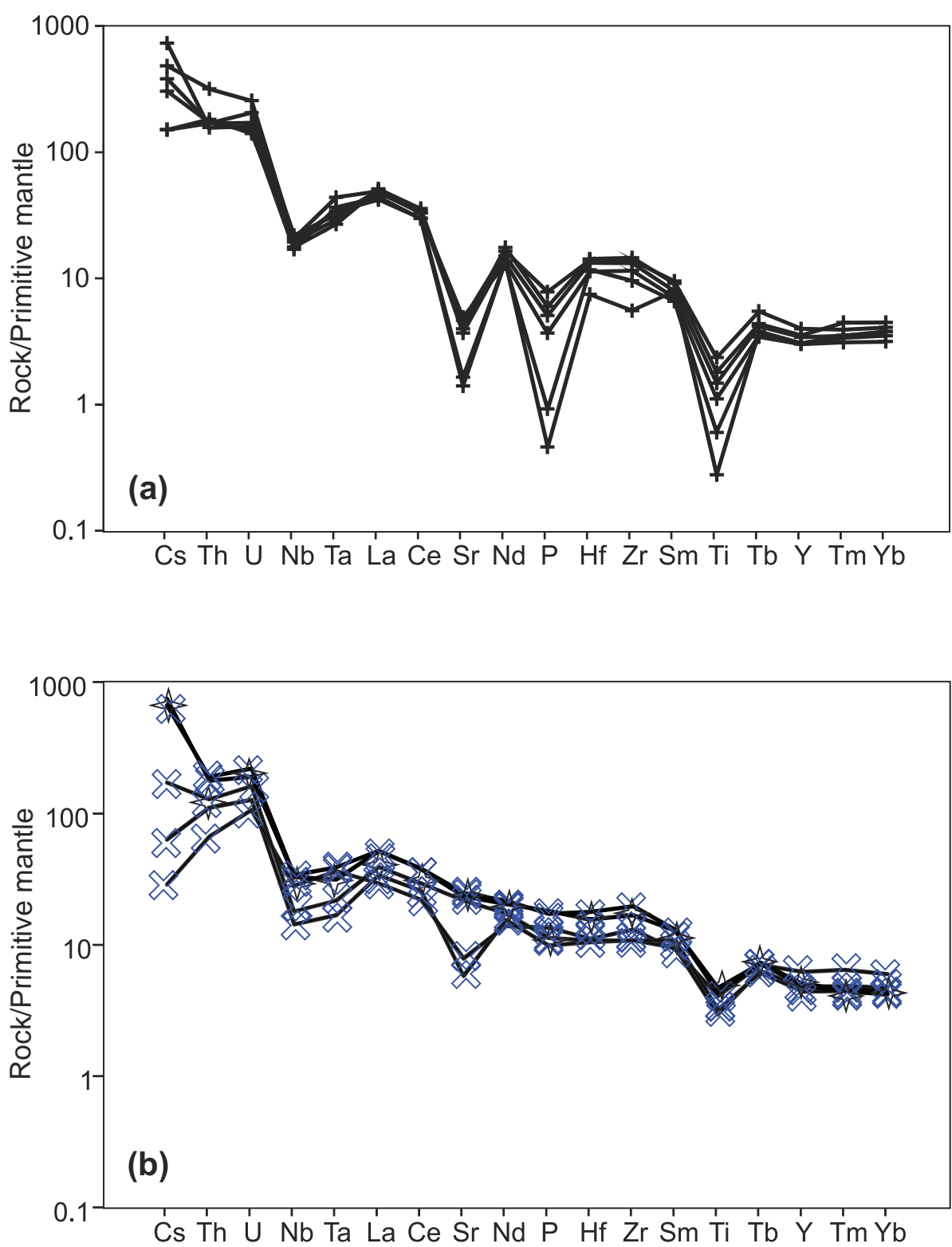

Fig. 12 Primary mantle-normalized (Sun and McDonough 1989) trace-element patterns of Kuh-e Dom dikes, acid (a) and intermediate-basic (b).

Many researchers proposed that the K-rich metasomatized lithospheric mantle is the main source of shoshonitic and mafic K-rich rocks in continental arcs (e.g. Meen and Eggler 1987; Lange and Carmichael 1991; Edwards et al. 1991; Wallace and Carmichael 1992). The enrichment of the mantle wedge in incompatible elements can be related to (Rock 1991): (1) involvement of an anomalous, metasomatized potassium-rich lithospheric mantle domain, or (2) recycling of a crustal component in subduction zones (e.g. Tatsumi and Eggins 1995; Jahn et al. 1999). Moreover, the presence of water-bearing minerals such as biotite and hornblende in the lithospheric mantle indicates that it had been metasomatized (Hawkesworth et al. 1990) as low Nb/U ratios (3-17) do (Griffin et al. 1988; O'Reilly and Griffin 1988; Maury et al. 1992). This is consistent with the previous experimental results (Edgar et al. 1976; Mengel and Green 1989). The experiments of Wyllie and Sekine (1982) demonstrated that reaction between hydrous fluids/melts and mantle peridotite produce hybrid mantle which could subsequently yield potassic melts (Jiang et al. 2005).

An important feature of subduction-related mantle metasomatism is the formation of hydrous mineral phase(s) phlogopite and/or amphibole (Beccaluva et al. 2004). Müller and Groves (1997) suggested that the likely mineral in the source is phlogopite for many potassic rocks, and that melting of a phlogopitebearing peridotite may produce potassium-rich parent magma. Zhang et al. (2008) argued that the presence of phlogopite or pargasitic hornblende in the source can lead to formation of K-rich magmas. Indeed, the experiments confirmed the stability of these potassium- and water-bearing minerals at $<30$ kbar and $\mathrm{T}<1050{ }^{\circ} \mathrm{C}$ (Edgar et al. 1976; Mengel and Green 1989).

Given the differences in compatibility relative to phlogopite and amphibole, the ratios of some incompatible elements can be used to constrain which hydrous phase existed in the melted lithospheric source (Furman and Graham 1999; Yang et al. 2004; Xie et al. 2006). For example, melts in equilibrium with phlogopite should show higher $\mathrm{Rb} / \mathrm{Sr}$ $(>0.1)$ and lower $\mathrm{Ba} / \mathrm{Rb}(<20)$ ratios than those from amphibole-bearing sources at the same degree of partial melting (Furman and Graham 1999). As Ba is partitioned into phlogopite to a greater extent than $\mathrm{Rb}$ (Ionov et al. 1997), partial melts, which leave phlogopite in the residue, will acquire a low $\mathrm{Ba} / \mathrm{Rb}$ ratio. On the other hand, significant removal of phlogopite from the residue by 
(higher degree) partial melting of the veins will tend to increase the $\mathrm{Ba} / \mathrm{Rb}$ ratios (Kurt et al. 2008).

Figure 14 shows that high-K samples from the Kuh-e Dom intermediate-basic dikes tend to display low Ba/ $\mathrm{Rb}$ and variably high $\mathrm{Rb} / \mathrm{Sr}$ ratios, which support a low-degree partial melting of phlogopite-bearing veined lithospheric mantle source. Moreover, the studied dike samples have constantly high $\mathrm{Yb}$ concentrations exceeding $1.8 \mathrm{ppm}$ and thus do not support amphibole as residual mineral phase in the mantle source (Guo et al. 2005). However, the degree of enrichment of middle REE relative to heavy REE such as $\mathrm{Yb}$ depends mainly on garnet existence as a residual phase during melting because HREE are preferentially retained by this mineral (Zhang et al. 2008). Hence, minor changes in $\mathrm{Sm} / \mathrm{Yb}$ ratio (1.3-2.7) in the studied intermediate-basic dikes underline lack of garnet in the mantle source.

Although phlogopite is not a typical mantle mineral and its presence in the upper mantle reveals compositional anomalies (Peccerillo 2003), it seems that a phlogopite-bearing lithospheric mantle metasomatized by subduction-related fluids or melts could be a potential melt source in our case (Conticelli and Peccerillo 1992). This small-scale heterogeneity is commonly considered to be due to the presence of a vein network permeating the upper-mantle peridotite (Foley 1992; Perini et al. 2004; Krmíček et al. 2011).

The veins are inferred to be rich in clinopyroxene together with accessory phases, such as phlogopite, titanite, and oxides, uncommon in normal peridotitic mantle. The low degrees of partial melting of such a veined phlogopite-bearing mantle would allow almost pure vein material to melt, generating highly potassic magmas. At higher degrees of melting, the same source would allow increasing amounts of lherzolite wallrock to enter the melt, generating magmas with lower enrichments in incompatible elements.

Potassium-bearing clinopyroxenes are known to exist in the deep mantle at $\mathrm{P}=5-7 \mathrm{GPa}$ and $\mathrm{T}>$ $1200^{\circ} \mathrm{C}$ (Tsuruta and Takahashi 1998). However, melting of deep mantle lithologies in the presence of residual K-clinopyroxene appears to be unlikely in case of the Kuh-e Dom dikes because (1) it would result in near horizontal trends of $\mathrm{CaO}$ versus $\mathrm{MgO}$ and $\mathrm{SiO}_{2}$ in the melt which are not observed, and (2) the HP-HT mantle melting conditions required for the generation of alkaline magma (1300-1500 ${ }^{\circ} \mathrm{C}$; Tsuruta and Takahashi 1998) would result in significantly larger volumes of melts which do not appear to exist in the studied region.

The range of variation in the trace element (LREE/ HFSE) characteristics of the magmas thus might be attributed to small-scale mantle heterogeneities, variable role for accessory phases in the residue (e.g., phlogopite and apatite) or the difference in the relative proportion

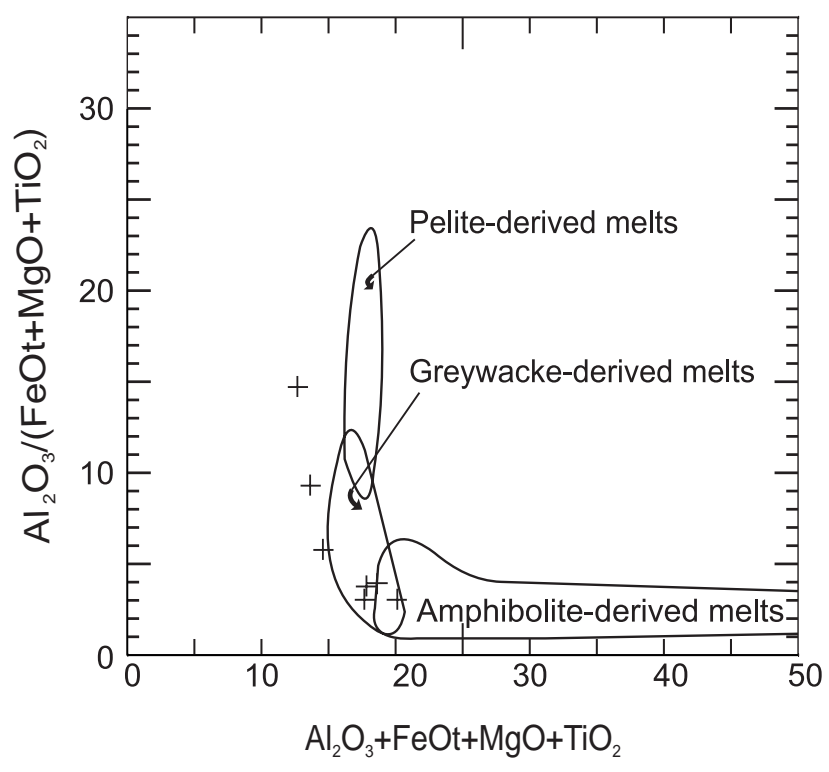

Fig. $13 \mathrm{Al}_{2} \mathrm{O}_{3}+\mathrm{FeOt}+\mathrm{MgO}+\mathrm{TiO}_{2}$ vs. $\mathrm{Al}_{2} \mathrm{O}_{3} /\left(\mathrm{FeOt}+\mathrm{MgO}+\mathrm{TiO}_{2}\right)($ Patiño Douce 1999) diagram indicating the partial melting of a quartzo-feldspathic crustal source.

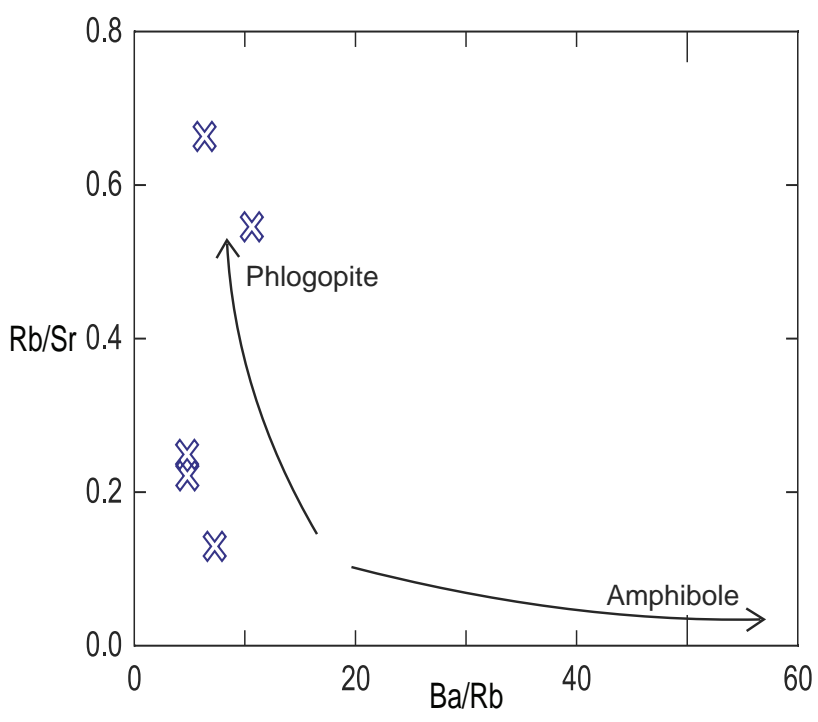

Fig. 14 Low $\mathrm{Ba} / \mathrm{Rb}$ and high $\mathrm{Rb} / \mathrm{Sr}$ ratios, showing phlogopite-bearing mantle source of intermediate-basic dikes (Furman and Graham 1999).

of veins and peridotitic wall-rock components, which contributed to the melt (i.e., degree of partial melting). The variable K-contents probably reflect melting of different amounts of phlogopite, or variable degree of partial melting of a phlogopite-bearing melt source.

\subsection{Tectonic setting}

Potassium-rich rocks are found in several tectonic environments, such as cratons, post-collisional setting, active orogenic belts and, to a lesser extent, oceanic intraplate 

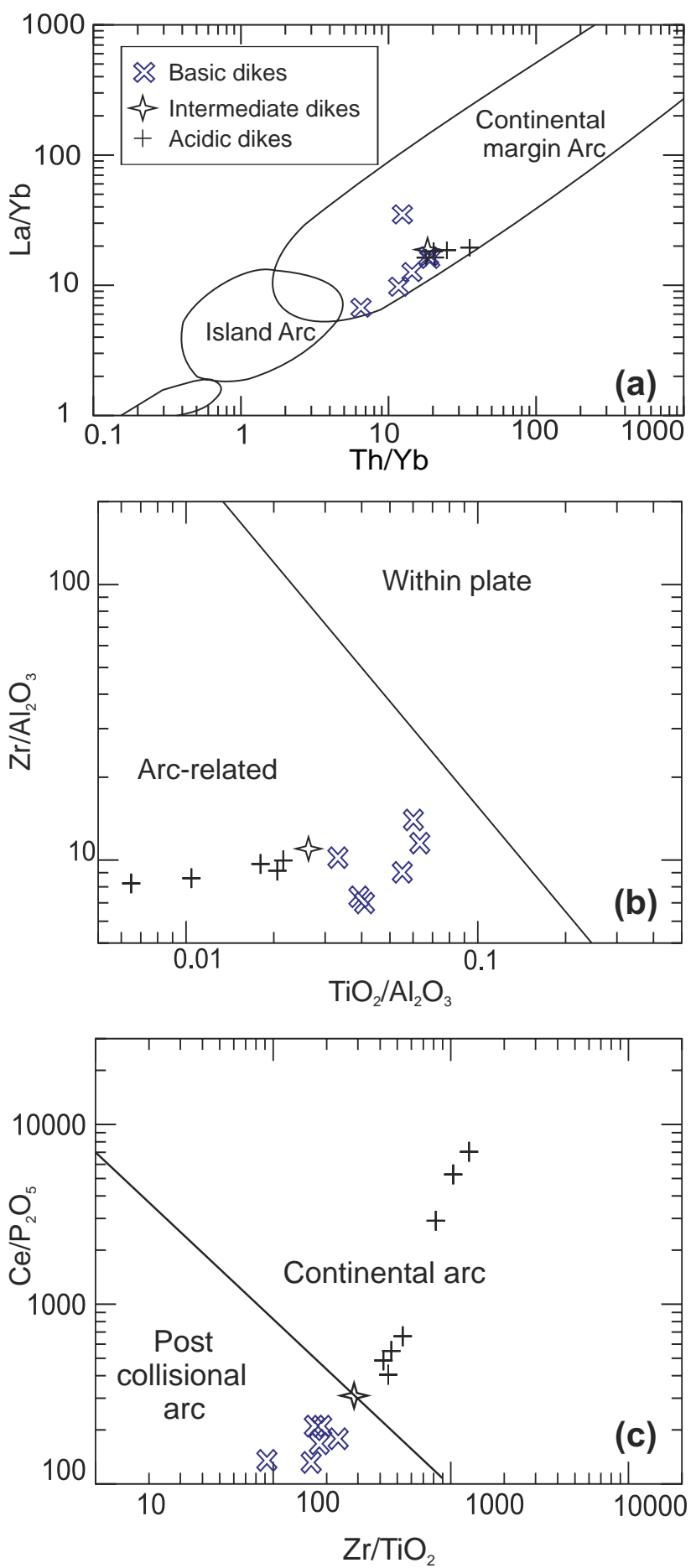

Fig. 15 Distribution of the selected samples in the geotectonic discrimination diagrams of Condie (1989) (a) and Müller and Groves (1997) (b-c).

settings (Müller and Groves 1997). Shoshonitic rocks are generally associated with many continental and oceanic arcs (Peccerillo 1992).

The studied dikes show enrichment in LILE and LREE, relative to HREE and HFSE, with negative anom- alies in Ta, Ti and $\mathrm{Nb}$. The average $\mathrm{Ba} / \mathrm{Nb}$ and $\mathrm{Rb} / \mathrm{Nb}$ ratios are 37.5 and 10.5 in acidic dikes and 62.5 and 7.6 in the intermediate-basic dikes, respectively. Such high values are typical of potassic igneous rocks formed at active continental margins (Müller and Groves 1997) because HFSE tend to remain in the subducted plate, whereas the LILE are easily transferred into the mantle wedge (Pearce 1983; Tatsumi and Eggins 1995). By comparison, these ratios are relatively low in the OIB ( 7.3 and $\sim 0.6$ ) and MORB ( 2.7 and $\sim 0.24$; Sun and McDonough 1989; Harangi 1994; Carmichael et al. 1996). Moreover, it is noted that the all Kuh-e Dom shoshonitic dikes plot within the fields of continental arcs in the $\mathrm{La} / \mathrm{Yb}-\mathrm{Th} /$ $\mathrm{Yb}$ tectonomagmatic discrimination diagram (Condie 1989; Fig 15a). The low Hf/Sm ratio ( 1) in the studied dikes also indicates a subduction-related orogenic setting (Lafleche et al. 1991). Finally, the $\mathrm{Zr} / \mathrm{Hf}$ ratios (27-37: acidic dikes, 33.6-42.6: intermediate-basic dikes) are consistent with potassic igneous rocks in continental-arc settings (Carmichael et al. 1996).

Müller and Groves (1997) suggested various discrimination diagrams for distinguishing between different tectonic settings of potassic rocks, especially active continental margins from post-collisional settings. All of Kuh-e Dom shoshonitic dike compositions classify as arc-related (Fig. 15b); in Fig 15c the silicic dikes fall in the continental-arc field while the intermediate-basic dikes seem post-collisional.

In summary, the combination of field, petrographic and geochemical data of the Kuh-e Dom shoshonitic dikes demonstrates that they formed at late stage of development of an active continental margin. This is in accord with previous reports on the petrogenesis of igneous rocks in the Urumieh-Dokhtar magmatic belt (Berberian and King 1981; Mohajjel et al. 2003; Shahabpour 2007). Indeed, recent tectono-magmatic syntheses have suggested that the Eocene magmatic event may have been a consequence of geometrically complex subduction of Neotethys oceanic crust underneath Central Iran. The acidic dikes which intruded the Kuh-e Dom pluton were emplaced in a transitional subduction to syn-collisional setting whereas the intermediate-basic dikes have developed after a short time gap, probably in the postcollisional setting (Technoexport 1981).

Recently, geologists have paid more attention to the dike swarms produced after the large-scale orogenic granite magmatism (Dini et al. 2002; Jiang et al. 2006), which have been termed the post-batholith dike swarms (Allen 2000) or post-orogenic complexes (Luo et al. 2006). It is proposed that such dike swarms are produced during the switch of the regional tectonic stress field from compression to extension. Therefore they could be marking the end of the orogeny similar to Taihangshan, Yanshan, Eastern Kunlun and Tianshan orogenic belts (Luo et al 2008). 


\section{Conclusions}

Based on petrographic and geochemical data from the Eocene Kuh-e Dom shoshonitic dikes, the following conclusions were reached:

1. All shoshonitic dikes can be subdivided into two groups: silicic (porphyric microgranodiorites, porphyric microgranites and aplites) and intermediate-basic (chemically ranging from phonolite to trachyandesite and basanite).

2. The silicic dikes are subaluminous and show shoshonitic affinity, with $\mathrm{Ti}, \mathrm{P}, \mathrm{Sr}$ and $\mathrm{Nb}$ depletion in Primitive-mantle normalized spiderplots indicating generation by partial melting of a quartzo-feldspathic source.

3. The intermediate-basic dikes have a metaluminous and shoshonitic nature. The high $\mathrm{Rb} / \mathrm{Sr}$ with low $\mathrm{Ba} / \mathrm{Rb}$ ratios document that their parental magma originated by partial melting of a phlogopite-bearing enriched mantle.

4. The mineral chemistry and whole-rock enrichments in LILE and LREE over HFSE and HREE, low Hf/ $\mathrm{Sm}$, as well as high $\mathrm{Ba} / \mathrm{Nb}, \mathrm{Rb} / \mathrm{Nb}$ and $\mathrm{Zr} / \mathrm{Hf}$ ratios indicate that Kuh-e Dom dikes formed during subduction of oceanic lithosphere beneath central Iran continent. The silicic dikes intruded during the late subduction and the intermediate-basic dikes soon thereafter.

Acknowledgements. This study is a synthesis of the Ph.D. thesis by F. Sarjoughian. Thanks are due to the University of Tehran for supporting this project by grants provided by the Research Council. Partial funding for this project was provided by the Research Office at the University of Payame Noor and we wish to acknowledge the generous support from all the staff of that office. We acknowledge Assoc. Prof. Janoušek, Dr. Krmíček and an anonymous reviewer for their constructive comments leading to important improvements in the manuscript.

\section{References}

Abdel-Rahman AM (1994) Nature of biotites from alkaline, calc-alkaline and peraluminous magmas. J Petrol 35: 525-541

Alavi M (1980) Tectonostratigraphic evolution of the Zagrosides of Iran. Geology 8: 144-149

Alavi M, Mahdavi MA (1994) Stratigraphy and structure of the Nahavand region in western Iran and their implications for the Zagros tectonics. Geol Mag 131: 43-47

Aldanmaz E, Pearce JA, Thirlwall MF, Mitchell JG (2000) Petrogenetic evolution of late Cenozoic, postcollision volcanism in western Anatolia, Turkey. J Volcanol Geotherm Res 102: 67-95
Allen CM (2000) Evolution of a post-batholith dike swarm in central coastal Queensland, Australia: arc-front to backarc? Lithos 51: 331-349

Beccaluva L, Bianchini G, Bonadiman C, Siena F, Vaccaro C (2004) Coexisting anorogenic and subduction-related metasomatism in mantle xenoliths from the Betic Cordillera (southern Spain). Lithos 75: 67-87

Berberian M, King GCP (1981) Towards a paleogeography and tectonic evolution of Iran. Can J Earth Sci 18: 210-265

Berberian F, Muir ID, Pankhurst RJ, Berberian M (1982) Late Cretaceous and early Miocene Andean-type plutonic activity in northern Makran and central Iran: J Geol Soc, London 139: 605-614

Carmichael ISE, Lange RA, Luhr JF (1996) Quaternary minettes and associated volcanic rocks of Mascota, western Mexico: a consequence of plate extension above a subduction modified mantle wedge. Contrib Mineral Petrol 124: 302-333

Chappell BW, White AJR (1974) Two contrasting granite types. Pac Geol 8: 173-174

Chappell BW, White AJR (1992) I and S-type granites in the Lachlan Fold Belt. Trans Roy Soc Edinb, Earth Sci 83: 1-26

Clemens JD, Wall VJ (1984) Origin and evolution of a peraluminous silicic ignimbrite suite: the Violet Town Volcanics. Contrib Mineral Petrol 88: 354-371

CondIE KC (1989) Geochemical changes in basalts and andesites across the Archean-Proterozoic boundary: identification and significance. Lithos 23: 1-18.

Condie KC, Latysh N, Van Schmus WR, Kozuch M, Selverstone J (1999) Geochemistry, Nd and Sr isotopes, and $\mathrm{U} / \mathrm{Pb}$ zircon ages of granitoid and metasedimentary xenoliths from the Navajo Volcanic Field, Four Corners area, Southwestern United States. Chem Geol 156: 95-133

Conticelli S, Peccerillo A (1992) Petrology and geochemistry of potassic and ultrapotassic volcanism in central Italy: petrogenesis and inferences on the evolution of the mantle sources. Lithos 28: 221-240

DeEr WA, Howie RA, Zussman J (1992) An Introduction to the Rock-Forming Minerals. Longman, London, pp 1-696

Dini A, InNocenti F, Rocchi S, Tonarini S, Westerman DS (2002) The magmatic evolution of the late Miocene laccolith-pluton-dike granitic complex of Elba Island, Italy. Geol Mag 139: 257-279

DROOP GTR (1987) A general equation for estimating $\mathrm{Fe}^{3+}$ in ferromagnesian silicates and oxides from microprobe analysis, using stoichiometric criteria. Mineral Mag 51: 431-437

Edgar AD, Green DH, HibBerson WO (1976) Experimental petrology of a highly potassic magma. J Petrol 17: 339-356

Edwards C, Menzies M, Thirlwall M (1991) Evidence from Muriah, Indonesia, for the interplay of supra subduction 
zone and intraplate processes in the genesis of potassic alkaline magmas. J Petrol 32: 555-592

Feeley TC, Cosca MA (2003) Time vs. composition trends of magmatism at Sunlight volcano, Absaroka Volcanic Province, Wyoming. Geol Soc Am Bull 115: 714-728

Foley SF (1992) Veins plus wall-rock melting mechanism in the lithosphere and the origin of potassic alkaline magmas. Lithos 28: 435-453

Furman T, Graham D (1999) Erosion of lithospheric mantle beneath the East African Rift system: geochemical evidence from the Kivu volcanic province. Lithos 48: 237-262

Griffin WL, O’Reilly SY, Stabel A (1988) Mantle metasomatism beneath western Victoria, Austria: II. isotopic geochemistry of Cr-diopside lherzolites and Al-augite pyroxenites. Geochim Cosmochim Acta 52: 449-459

Guo Z, Hertogen J, Liu J, Pasteels P, Boven A, Punzalan L, He H, Luo X, Zhang W (2005) Potassic magmatism in Western Sichuan and Yunnan provinces, SE Tibet, China: petrological and geochemical constraints on petrogenesis. J Petrol 46: 33-78

HARANGI S (1994) Geochemistry and petrogenesis of the early Cretaceous continental rift type volcanic rocks of the Mecsek Mountains, South Hungary. Lithos 33: 303-321

Hastie AR, Kerr AC, Pearce JA Mitchell SF (2007) Classification of altered volcanic island arc rocks using immobile trace elements: development of the Th-Co discrimination diagram. J Petrol 48: 2341-2357

Hawkesworth CJ, Kempton PD, Rogers NW, Ellam RM, Calsteren PW (1990) Continental mantle lithosphere, and shallow level enrichment processes in the Earth's mantle. Earth Planet Sci Lett 96: 256-268

Hawthorne FC, OBERti R (2007) Classification of the amphiboles. In: Hawthorne FC, Oberti R, Della Venturae G, Mottana A (eds) Amphiboles: Crystal Chemistry, Occurrence and Health Issues. Mineralogical Society of America and Geochemical Society Reviews in Mineralogy and Geochemistry 67: 55-88

Ionov DA, GRIFFIN WI, O’ReILLY SY (1997) Volatile-bearing minerals and lithophile trace elements in the upper mantle. Chem Geol 141: 153-184

JaHN BM, Wu F, Lo CH, TsaI CH (1999) Crust-mantle interaction induced by deep subduction of the continental crust: geochemical and $\mathrm{Sr}-\mathrm{Nd}$ isotopic evidence from post-collisional mafic-ultramafic intrusions of the northern Dabie Complex, central China. Chem Geol 157: 119-146

JiAng YH, JiAng SY, Ling HF, Zhou XR, Rui XJ, Yang WZ (2002) Petrology and geochemistry of shoshonitic plutons from the western Kunlun Orogenic Belt, Xinjiang, northwestern China: implications for granitoid geneses. Lithos 63: 165-187
Jiang YH, Ling HF, Jiang SY, Fan HH, Shen WZ, Ni P (2005) Petrogenesis of a Late Jurassic peraluminous volcanic complex and its high-Mg, potassic, quenched enclaves at Xiangshan, Southeast China. J Petrol 46: 1121-1154

Jiang YH, Jiang SY, Zhao KD, Ling HF (2006) Petrogenesis of Late Jurassic Qianlishan granites and mafic dikes, southeast China: implications for a back-arc extension setting. Geol Mag 143: 457-474

JoPLIN GA (1965) The problem of potash-rich basaltic rocks. Mineral Mag 34: 266-275

JopLIN GA (1968) The shoshonite association: a review. J Geol Soc Aust 15: 275-294

KelLer J (1974) Petrology of some volcanic rock series of the Aeolian Arc, southern Tyrrhenian Sea, calc-alkaline and shoshonitic associations. Contrib Mineral Petrol 46: 29-47

Krmíčer L, Cempírek J, Havlín A, Pěichystal A, Houzar S, KRMíčKovÁ M, GADAS P (2011) Mineralogy and petrogenesis of a Ba-Ti-Zr-rich peralkaline dyke from Šebkovice (Czech Republic): recognition of the most lamproitic Variscan intrusion. Lithos 121: 74-86

Kurt MA, Alpaslan MC, GönCÜoĜlu MC, Temel A (2008) Geochemistry of late stage medium to high-K calcalkaline and shoshonitic dykes in the Ulukişla Basin (Central Anatolia, Turkey): petrogenesis and tectonic setting. Geochem Int 46: 1145-1163

Lafleche MR, Dupuy C, Dostal J (1991) Archaean orogenic ultrapotassic magmatism: an example from the Southern Abitibi Greenstone Belt. Precambr Res 52: 71-96

Lange RA, Carmichael ISE (1991) A potassic volcanic front in Western Mexico - the lamprophyric and related lavas of San Sebastian. Geol Soc Am Bull 103: 928-940

LE BAS MJ (1962) The role of aluminum in igneous clinopyroxenes with relation to their parentage. Amer J Sci 260: 267-288

Leake BE, Woolley AR, Arps CES, Birch WD, Gilbert MC, Grice JD, Hawthorne FC, Кato A, Кisch HJ, Krivovichev VG, Linthout K, Laird J, Mandarino J, Maresch WV, Nickel EH, Rock NMS, Schumacher JC, Smith DC, Stephenson NCN, Ungaretti L, Whittaker EJW, Youzhi G (1997) Nomenclature of amphiboles. Report of the Subcommittee on Amphiboles of the International Mineralogical Association Commission on New Minerals and Mineral Names. Eur J Mineral 9: 623-651

Liégeois J-P, Navez J, Hertogen J, Black R (1998) Contrasting origin of post-collisional high-K calc-alkaline and shoshonitic versus alkaline and peralkaline granitoids. The use of sliding normalization. Lithos 45:1-28

Luo ZH, Wei Y, Xin HT, Zhan HM, Ke S, Li WT (2006) Petrogenesis of the post-orogenic dike complex - constraints to lithosphere delamination. Acta Petrol Sin 22: 1672-1684 
Luo ZH, Lu X, Wang B, Chen B, Huang F, Yang Z, Wang Y (2008) Postorogenic dike complexes and implications for metallogenesis. Earth Sci Front 15: 1-12

Maury RC, Defant MJ, Joron JL (1992) Metasomatism of the subarc mantle inferred from trace elements in Philippine xenoliths. Nature 360: 661-663

Meen JK (1987) Formation of shoshonites from calc alkaline basaltic magma, geochemical and experimental constraints from the type locality. Contrib Mineral Petrol 97: 333-351

MeEn JK, EgGLeR DH (1987) Petrology and geochemistry of the Cretaceous Independence Volcanic Suite, Absaroka Mountains, Montana: clues to the composition of the Archean sub-Montanan mantle. Geol Soc Am Bull 98: 238-247

Mengel K, Green DH (1989) Stability of amphibole and phlogopite in metasomatized peridotite under watersaturated and water-undersaturated conditions. In: Ross J (ed) Kimberlites and Related Rocks. Their Occurrence, Origin and Emplacement. Geological Society of Australia Special Publication 14: 571-581

Mohajel M, Fergusson CL, Sahandi MR (2003) Cretaceous-Tertiary convergence and continental collision, Sanandaj-Sirjan Zone, Western Iran: J Asian Earth Sci 21: 397-412

Molina J, Scarrow J, Montero PG, Bea F (2009) High-Ti amphibole as a petrogenetic indicator of magma chemistry: evidence for mildly alkalic-hybrid melts during evolution of Variscan basic-ultrabasic magmatism of Central Iberia. Contrib Mineral Petrol 158: 69-98

Morrison GW (1980) Characteristics and tectonic setting of the shoshonite rock association. Lithos 13: 97-108

Morimoto N (1988) Nomenclature of pyroxenes. Mineral Petrol 39: 55-76

Müller D, Forrestal P (1998) The shoshonite porphyry $\mathrm{Cu}-\mathrm{Au}$ association at Bajo de la Alumbrera, Catamarca Province, Argentina. Mineral Petrol 64:47-64

Müller D, Groves DI (1997) Potassic Igneous Rocks and Associated Gold-Copper Mineralization. $3^{\text {rd }}$ edition, Springer-Verlag, Berlin, pp 1-238

Nachit H, IвнI A, AвiA ELH, Ohoud MB (2005) Discrimination between primary magmatic biotites, re-equilibrated biotites and neoformed biotites. C R Acad Sci Geosci 337: $1415-1420$

O’Reilly SY, GRiffin WL (1988) Mantle metasomatism beneath western Victoria, Austria: I. Metasomatic processes in Cr-diopside lherzolites. Geochim Cosmochim Acta 52: 433-447

Patiño Douce AE, McCarthy TC (1998) Melting of crustal rocks during continental collision and subduction. In: Hacker BR, Liou JG (eds) When Continents Collide: Geodynamics and Geochemistry of Ultrahigh-Pressure Rocks. Dordrecht: Kluwer Academic, pp 27-55

Patiño Douce AE (1999) What do experiments tell us about relative contributions of crust and mantle to the origin of granitic magmas? In: Castro A, Fernández C, VignerESSE JL (eds) Understanding Granites: Integrating New and Classical Techniques: J Geol Soc, London, Spec Publ 168: 55-75

Pearce JA (1982) Trace element characteristics of lavas from destructive plate boundaries. In: THORPE RS (ed) Andesites. Wiley, New York, pp 525-548

Pearce JA (1983) Role of subcontinental lithosphere in magma genesis at active continental margins. In: HAwKESworth CJ, Norry MJ (eds) Continental Basalts and Mantle Xenoliths. Shiva, Nantwich, pp 230-249

Peccerillo A (1992) Potassic and ultrapotassic rocks: compositional characteristics, petrogenesis, and geologic significance. Episodes 15: 243-251

Peccerillo A (2003) Plio-Quaternary Magmatism in Italy. Episodes 26: 222-226

Peccerillo A, Taylor SR (1976) Geochemistry of Eocene calc-alkaline volcanic rocks from the Kastamonu area, Northern Turkey. Contrib Mineral Petrol 58: 63-81

Perini G, Ponticelli S, Francalanci L, Davidson JP (2004) Magmatological evolution and genesis of magmas from Vico volcano, Central Italy: multiple differentiation pathways and variable parental magmas. J Petrol 45: 139-182

Pouchou JL, Pichoir F (1985) "PAP" $\varphi(\rho z)$ correction procedure for improved quantitative microanalysis. In: Armstrong JT (eds) Microbeam Analysis. San Francisco Press, San Francisco, pp 104-106

RichARD LR (1995) MinPet: Mineralogical and petrological data processing system, version 2.02. MinPet Geological Software, Quebec, Canada

Ridolfi F, Puerini M, Renzulli A, Menna M, Toulkeridis T (2008) The magmatic feeding system of El Reventador volcano (Sub-Andean zone, Ecuador) constrained by texture, mineralogy and thermobarometry of the 2002 erupted products. J Volcanol Geotherm Res 176: 94-106

Rieder M, Cavazzini G, Dyakonov YS, Frank-KamenetskiI VA, Gottardi G, Guggenheim S, Koval P, Müller G, Neiva AMR, Radoslovich EW, Robert JL, SAssi FP, Takeda H, Weiss Z, Wones DR (1998) Nomenclature of the micas. Canad Mineral 36: 905-912

RoBerts MP, Clemens JD (1993) Origin of high-potassium, calc-alkaline, I-type granitoids. Geology 21: 825-828

Robinson P, Spear FS, Schumacher JC, Laird J, Klein C, Evans BW, Doolan BL (1981) Phase relations of metamorphic amphiboles: natural occurrence and theory. Mineralogical Society of America Reviews in Mineralogy, pp 1-213

Rock NMS (1991) Lamprophyres. Blackie, London, pp $1-285$

Rollinson H (1993) Using Geochemical Data: Evaluation, Presentation, Interpretation. Longman Scientific and Technical, New York, pp 1-352

Sarjoughian F (2007) Petrology and Geochemistry of Kuh-e Dom Intrusive (North Ardestan). Unpublished 
MSc. Thesis, University of Tehran, Iran, pp 1-138 (in Persian)

SarJoughian F (2012) Nature of the Kuh-E Dom Plutonism (NE Ardestan), Geological Events and Its Magmatic Evolution. Unpublished Ph.D Thesis, University of Tehran, Iran, pp 1-220 (in Persian)

Seghedi I, Downes H, Szakács A, Mason PRD, Thirlwall MF, Roşu E, Pécskay Z, Márton E, Panaiotu C (2004) Neogene-Quaternary magmatism and geodynamics in the Carpathian-Pannonian region: a synthesis. Lithos 72: 117-146

SHAHABPour J (2007) Island-arc affinity of the Central Iranian volcanic belt. J Asian Earth Sci 30: 652-665

Sun SS, McDonough WF (1989) Chemical and isotopic systematics of oceanic basalts; implications for mantle composition and processes. In: SAunders AD, Norry MJ (eds) Magmatism in the Ocean Basins. Geological Society of London Special Publications 42: 313-34

Tatsumi Y, Eggins S (1995) Subduction Zone Magmatism. Blackwell, Cambridge, pp 1-211

Technoexport (1981) Detail geology prospecting in the Anarak Area Central Iran. Geological Survey of Iran, Report N. 9, Tehran, pp 1-154

Tsuruta K, TAKAHASHi E (1998) Melting study of an alkali basalt JB-1 up to $12.5 \mathrm{GPa}$ : behavior of potassium in the deep mantle. Phys Earth Planet Inter 107: 119-130

Turner S, Arnaud N, Liu J, Rogers N, Hawkesworth C, Harris N, Kelley S, Van Calsteren P, Deng W (1996) Postcollision, shoshonitic volcanism on the Tibetan Plateau: implications for convective thinning of the lithosphere and the source of ocean island basalts. J Petrol 37: 45-71

VERnON RH (2004) A Practical Guide to Rock Microstructure. Cambridge University Press, Cambridge, pp 1-594

Wallace P, Carmichael ISE (1992) Alkaline and calcalkaline lavas near Los Volcanes, Jalisco, Mexico - geochemical diversity and its significance in volcanic arcs. Contrib Mineral Petrol 111: 423-439

WANG D, Zhou J, Qiu J (1991) The research status of shoshonitic series rocks. J Nanjing Univ (Earth Science) 4: 321-328
White AJR, Chappell BW (1983) Granitoid types and their distribution in the Lachlan Fold Belt, southeastern Australia. In: RodDick JA (ed) Circum-Pacific Plutonic Terranes. Geological Society of America, Memoirs 159: 21-34

Winchester JA, Floyd PA (1977) Geochemical discrimination of different magma series and their differentiation products using immobile elements. Chem Geol 20: 325-344

Wyborn D, Chappell BW, Johnston RM (1981) Three Stype volcanic suites from the Lachlan Fold Belt, southeast Australia. J Geophys Res 86: 10335-10348

Wyllie PJ, SEKINE T (1982) The formation of mantle phlogopite in subduction zone hybridization. Contrib Mineral Petrol 79: 375-380

Xie C, Zhu J, Ding S, Zhang Y, Fu T, Li Z (2006) Identification of Hercynian shoshonitic intrusive rocks in central Hainan Island and its geotectonic implications. Chinese Sci Bull 51: 2507-2519

Yang JH, Chung SL, Zhai MG, Zhou XH (2004) Geochemical and $\mathrm{Sr}-\mathrm{Nd}-\mathrm{Pb}$ isotopic compositions of mafic dikes from the Jiaodong Peninsula, China: evidence for vein-plus-peridotite melting in the lithospheric mantle. Lithos 73: 145-160

Yardley BWD, VAlLey JW (1997) The petrologic case for a dry lower crust. J Geophys Res 102: 12173-12185

YAvUZ F (2003) Evaluating micas in petrologic and metallogenic aspect: I - definitions and structure of the computer program MICA ${ }^{+}$. Comput Geosci 29: 1203-1213

ZEN E (1988) Tectonic significance of high pressure plutonic rocks in the Western Cordillera of North America. In: ERnst WG (ed) Metamorphism and Crustal Evolution of the Western United States. Prentice-Hall, New Jersey pp 41-67

Zhang Z, Xiao X, Wang J, Wang Y, Kusky TM (2008) Postcollisional Plio-Pleistocene shoshonitic volcanism in the western Kunlun Mountains, NW China: geochemical constraints on mantle source characteristics and petrogenesis. J Asian Earth Sci 31: 379-403 